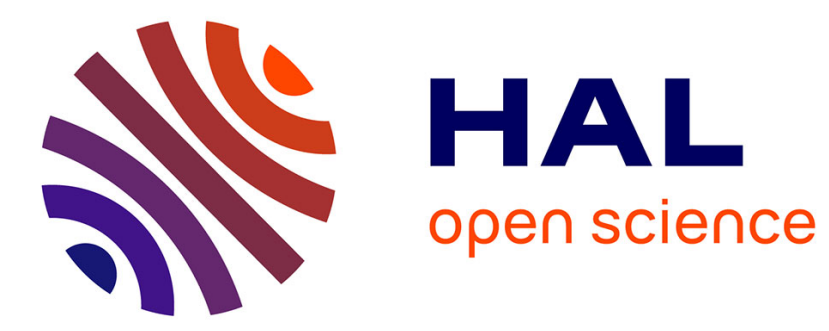

\title{
Air entrainment and bubble statistics in breaking waves
}

Luc Deike, W. Kendall Melville, Stéphane Popinet

\section{To cite this version:}

Luc Deike, W. Kendall Melville, Stéphane Popinet. Air entrainment and bubble statistics in breaking waves. Journal of Fluid Mechanics, 2016, 801, pp.91-129. 10.1017/jfm.2016.372 . hal-01361280

\section{HAL Id: hal-01361280 \\ https://hal.science/hal-01361280}

Submitted on 7 Sep 2016

HAL is a multi-disciplinary open access archive for the deposit and dissemination of scientific research documents, whether they are published or not. The documents may come from teaching and research institutions in France or abroad, or from public or private research centers.
L'archive ouverte pluridisciplinaire HAL, est destinée au dépôt et à la diffusion de documents scientifiques de niveau recherche, publiés ou non, émanant des établissements d'enseignement et de recherche français ou étrangers, des laboratoires publics ou privés. 


\title{
Air entrainment and bubble statistics in breaking waves
}

\author{
Luc Deike ${ }^{1} \dagger$, W. Kendall Melville ${ }^{1}$ and Stéphane Popinet ${ }^{2}$. \\ ${ }^{1}$ Scripps Institution of Oceanography, University of California San Diego, La Jolla, California \\ ${ }^{2}$ Sorbonne Universités, UPMC Univ Paris 06, CNRS, UMR 7190 Institut Jean Le Rond \\ d'Alembert, F-75005 Paris, France.
}

(Received 5 July 2016)

We investigate air entrainment and bubble statistics in three-dimensional breaking waves through novel direct numerical simulations of the two-phase air-water flow, resolving the length scales relevant for the bubble formation problem, the capillary length and the Hinze scale. The dissipation due to breaking is found to be in good agreement with previous experimental observations and inertial-scaling arguments. The air-entrainment properties and bubble-size statistics are investigated for various initial characteristic wave slopes. For radii larger than the Hinze scale, the bubble size distribution, can be described by $N(r, t)=B\left(V_{0} / 2 \pi\right)(\varepsilon(t-\Delta \tau) / W g) r^{-10 / 3} r_{m}^{-2 / 3}$ during the active breaking stages, where $\varepsilon(t-\Delta \tau)$ is the time dependent turbulent dissipation rate, with $\Delta \tau$ the collapse time of the initial air pocket entrained by the breaking wave, $W$ a weighted vertical velocity of the bubble plume, $r_{m}$ the maximum bubble radius, $g$ gravity, $V_{0}$ the initial volume of air entrained, $r$ the bubble radius and $B$ a dimensionless constant. The active breaking time-averaged bubble size distribution is described by $\bar{N}(r)=B(1 / 2 \pi)\left(\epsilon_{l} L_{c} / W g \rho\right) r^{-10 / 3} r_{m}^{-2 / 3}$, where $\epsilon_{l}$ is the wave dissipation rate per unit length of breaking crest, $\rho$ the water density and $L_{c}$ the length of breaking crest. Finally, the averaged total volume of entrained air, $\bar{V}$, per breaking event can be simply related to $\epsilon_{l}$ by $\bar{V}=B\left(\epsilon_{l} L_{c} / W g \rho\right)$, which leads to a relationship to a characteristic slope, $S$, of $\bar{V} \propto S^{5 / 2}$. We propose a phenomenological turbulent bubble break-up model, based on earlier models and the balance between mechanical dissipation and work done against buoyancy forces. The model is consistent with the numerical results and existing experimental results.

\section{Introduction}

\subsection{The broader context}

Surface wave breaking plays an important role in the coupling between the atmosphere and the ocean from local weather to global-climate scales. In the absence of wave breaking, the direct transport between the atmosphere and the ocean is through slow molecular diffusion and conduction processes. In contrast, when a wave breaks, the surface may experience dramatic changes, with a jet forming and plunging back to the surface, ejecting spray and entraining air into the ocean. For weaker breaking the surface may roll over itself down the front of the wave, while still entraining bubbles.

Breaking is a transitional flow process from the laminar fluid dynamics of classical surface waves to a two-phase turbulent flow. Thus wave breaking limits the height of surface waves, transfers momentum from waves to currents and significantly enhances the transfer of heat, water vapour, marine aerosols and gases between the atmosphere and

$\dagger$ Email address for correspondence: ldeike@ucsd.edu 
the ocean (Memery \& Merlivat 1985; Farmer et al. 1993; Melville 1996). Approximately $30 \%$ of the $\mathrm{CO}_{2}$ released into the atmosphere is taken up by the ocean (Rhein et al. 2013), much from the entrainment and dissolution of bubbles by breaking and the associated mixing (Monahan \& Dam 2001). Once in solution, $\mathrm{CO}_{2}$ forms carbonic acid, the source of ocean acidification, which has such an adverse effect on shell-forming marine animals. Breaking impact forms spray and aerosols, while small bubbles may be dissolved into the water column, larger bubbles entrained by breaking rise back to the surface and collapse. This generates spray, which is transported into the atmosphere and ultimately evaporates leaving water vapour, important for the thermodynamics of the atmosphere, and salt crystals that affect the radiative balance of the atmosphere and form cloud condensation nuclei (Andreas et al. 1995; de Leeuw et al. 2011; Veron 2015).

For all these reasons, improvements in our understanding of the ocean, atmosphere and climate systems requires a detailed understanding of the physics of air entrainment and subsequent bubble generation. Due to the complex nature of the breaking process, a coupled two-phase turbulent flow, a detailed understanding of the dynamical and statistical properties of the generated bubbles has been elusive.

\subsection{Bubble size distribution, models and observations}

The bubble size distribution is the most important characteristic of the bubble formation process since one can retrieve the bubble cloud properties, volume, energy, and penetration depth from its moments. Garrett et al. (2000) introduced the most widely used model for the bubble size distribution. It relies on a steady model of a turbulent break-up cascade, assuming the size distribution per unit volume $\mathcal{N}(r)$, with $r$ the bubble radius, to depend only on the local (time-averaged) turbulent dissipation rate $\bar{\varepsilon}$, the bubble radius $r$ and the source of bubbles, i.e. the constant air flow rate per unit volume of water $Q$. The steady assumption requires $\mathcal{N}(r) \propto Q$, then dimensional analysis leads to:

$$
\mathcal{N}(r) \propto Q \bar{\varepsilon}^{-1 / 3} r^{-10 / 3} .
$$

Note that Garrett et al. (2000) consider the bubble size distribution per unit volume $\mathcal{N}(r)$, which is physically equivalent to $N(r)$ the bubble size distribution $(\mathcal{N}(r) d r$ and $N(r) d r$ are respectively the per unit volume, and absolute number of bubbles of radii between $r$ and $r+d r$ ). If one considers $N(r)$, Eq. 1.1 is unchanged, except $Q$ is now the absolute air flow rate.

The related turbulent break-up model assumes an inertial subrange and a direct cascade process: air is injected at large scales (large bubbles) by the entrainment process and turbulent fluctuations break them into smaller bubbles. The cascade process ends at the scale where surface tension prevents further bubble break-up, the Hinze scale (Hinze 1955):

$$
r_{H}=\mathcal{C}(\gamma / \rho)^{3 / 5} \bar{\varepsilon}^{-2 / 5},
$$

where $\gamma$ is the surface tension, $\rho$ the water density and $\mathcal{C}$ is a dimensionless constant which has a value of approximately 0.5 (Martinez-Bazan et al. 1999; Garrett et al. 2000; Deane \& Stokes 2002).

Several experimental studies (Loewen et al. 1996; Terrill et al. 2001; Deane \& Stokes 2002; Leifer \& de Leeuw 2006; Rojas \& Loewen 2007; Blenkinsopp \& Chaplin 2010) have identified a bubble size distribution following a power law of the bubble radius $N(r) \propto r^{-m}$ with $m \in[2.5: 3.5]$, roughly compatible with Eq. (1.1) when considering the difficulty of the experiments. Figure 1 shows the bubble size distribution from various laboratory studies, using different measurement methods: cameras and bubble interface detection technique (Deane \& Stokes 2002), fiber optical probes (Rojas \& Loewen 2007; 


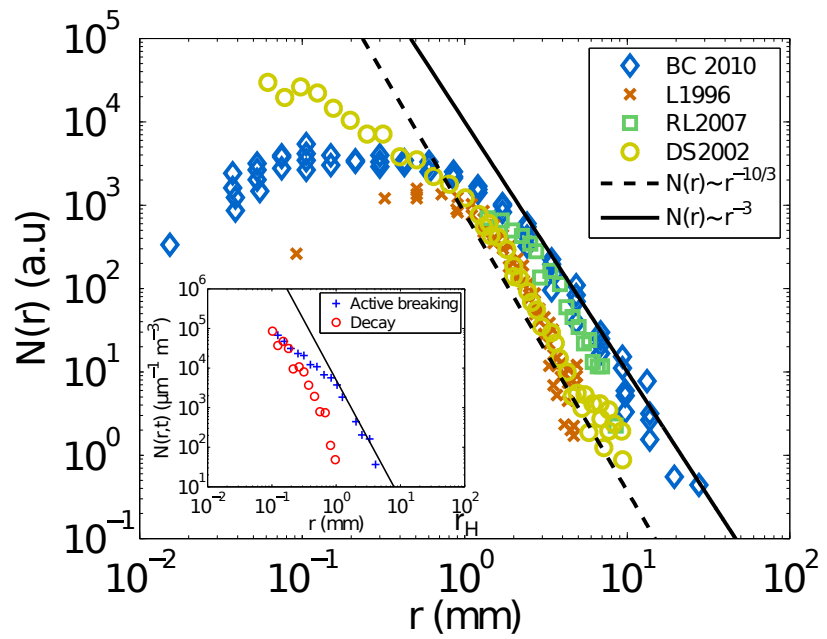

Figure 1: Bubble size distributions $N(r)$, measured during various breaking waves laboratory experiment, in the existing literature. Dashed line is $N(r) \propto r^{-10 / 3}$ and solid line is $N(r) \propto r^{-3}$. Size distributions are given in arbitrary units due to various normalizations in the original papers. $(\diamond)$ BC2010 stands for Blenkinsopp \& Chaplin $(2010),(\times)$ L1996 for Loewen et al. (1996), ( $\square$ ) RL2007 for Rojas \& Loewen (2007) and (o) DS2002 for Deane \& Stokes (2002). (Inset) Experimental bubble size distributions $N(r, t)$ from Deane \& Stokes (2002), during the active breaking stage $(+)$ and later during the decay (o). Solid line is $N(r, t) \propto r^{-3}$. $N(r, t)$ is much steeper once the active breaking stage is finished.

Blenkinsopp \& Chaplin 2010) or acoustic instruments (Loewen et al. 1996). From Figure 1 it is hard to make a clear statement on the exact value of the power law exponent $m$. Data from Deane \& Stokes (2002) and Loewen et al. (1996) are closer to the $m=10 / 3$ value while data from more recent experiments shows values closer to $m=3$ (Rojas \& Loewen 2007; Blenkinsopp \& Chaplin 2010). Moreover, the experiments by Deane \& Stokes (2002) and Blenkinsopp \& Chaplin (2010) give very different shapes for $N(r)$ below the Hinze scale.

One of the difficulties in properly identifying the exponent $m$ is the rapid time variations of the bubble size distribution. As discussed by Deane \& Stokes (2002) and shown in Figure 1 (inset), the scaling $N(r) \propto r^{-m}$, with $m \in[2.5: 3.5]$, is only valid during the active breaking time, which is around one wave period. Later on, the bubble size distribution is found to be much steeper. Therefore, it is possible that the different values for $m$ found in the literature are related to the time of observation and the time of averaging used to calculate $N(r)$. Therefore a more complete description of the time evolution of the bubble size distribution during the breaking event and during the rise of the bubbles is needed.

Indeed, the model from Garrett et al. (2000) (Eq. (1.1)), while undoubtelly correct for constant $Q$, does not describe the temporal evolution in which the bubble size distribution experiences very rapid change (Terrill et al. 2001; Deane \& Stokes 2002). Moreover, measurements of the volume of entrained air have shown that $Q$ is not a constant parameter in this problem (Lamarre \& Melville 1991; Blenkinsopp \& Chaplin 2007). Finally the dependence on $\bar{\varepsilon}$ and $Q$ in Eq. 1 has never been validated and raises a concern. In breaking waves, even if there were a constant $Q$ for some time, it would almost certainly 
depend on $\bar{\varepsilon}$, in which case we might expect that $Q \bar{\varepsilon}^{-1 / 3} \propto \bar{\varepsilon}^{n}$, with $n>0$. Thus, understanding the link between these two variables is crucial and will clarify the relationship between the kinematics and dynamics of the flow (e.g. dissipation, air entrainment rate) and the integral quantities, through the moments of the size distribution.

Baldy (1993) proposed another model for bubble generation by breaking waves, also based on a turbulent break-up scenario and describes $s(r, t)$ the bubble size distribution per unit time, per unit mass per unit ocean surface area, assuming adiabatic evolution, i.e. the time scale of bubble fragmentation is much faster than the other processes at play. Considering that $s(r, t)$ is given by the balance of the local time dependent turbulent dissipation rate $\varepsilon(t)$ and characteristic surface tension energy of a single bubble $e_{s} \propto$ $\gamma r^{2} / \rho$, Baldy (1993) found by dimensional analysis:

$$
s(r, t) \propto \frac{\varepsilon(t)}{(\gamma / \rho) r^{2}} \propto \varepsilon(t)\left(\frac{\gamma}{\rho}\right)^{-1} r^{-2} .
$$

This model is appealing because it considers only the bubble energy and the turbulent break-up dissipation rate while Garrett et al. (2000) had to introduce the constant air flow rate $Q$ to close their dimensional argument. Since this model is adiabatic in time, the measurable time-averaged bubble size distribution $N(r)$ is going to be $N(r) \propto r^{-2}$. However, as discussed before, experimental results suggest that $N(r) \propto r^{-m}$, with $m \in$ $[2.5: 3.5]$, which is quite different from the above result (Eq. 1.3). Note also that this model is based on an inertial subrange hypothesis without indications of the scale of the smallest bubble, so the role of the Hinze scale in this description is therefore not clear.

On the other hand, the Garrett et al. (2000) model predicts $m=10 / 3$, which, within the scatter, is in agreement with experimental data. An important difference between the models from Baldy (1993) and Garrett et al. (2000) concerns the treatment of surface tension. Baldy (1993) considers surface tension energy as the key parameter to balance the turbulent break-up of the bubbles within the turbulent inertial subrange, while Garrett et al. (2000) neglect surface tension for bubbles of radius larger than the Hinze scale, assuming that surface tension is the process that stops the turbulent break-up at small scale.

\subsection{Numerical Simulations}

Besides laboratory and field work, numerical simulations of the two-phase breaking wave flow have recently become available through improvements in numerical schemes and increases in computational capacity, but they still remain very challenging. Two approaches exist: large eddy simulations (LES), with subgrid-scale turbulent and bubbly-flow models and direct numerical simulations (DNS). While LES solves the large scales directly, strong asumptions are needed for the subgrid-scale turbulent bubbly flow closures, e.g. assuming a bubble size distribution (Shi et al. 2010; Liang et al. 2011, 2012; Derakhti \& Kirby 2014). In contrast, DNS is an appealing tool since no parametrizations are used to solve the multi-phase flow. The DNS has been limited to two-dimensional evolution of periodic unstable waves with relatively small wavelengths, providing numerical data on wave dissipation and the splashing processes (Chen et al. 1999; Song \& Sirviente 2004; Iafrati 2011; Deike et al. 2015). Three dimensional simulations of breaking waves have recently become available, both DNS (Fuster et al. 2009) and LES (Derakhti \& Kirby 2014; Lubin \& Glockner 2015). They are indeed necessary to investigate bubble and spray formation, which are fundamentally three-dimensional processes. We present here a DNS study of air entrainment and the bubble statistics in three-dimensional breaking waves, for various breaking intensities. The focus is on air entrainment while predictions of spray formation will be considered in future work. 


\subsection{Outline}

We present novel DNS of the two-phase air-water flow permitting solutions for fully three-dimensional breaking waves including the air entrainment and subsequent bubble formation. We investigate both the time evolution of the air entrainment, through the total volume of entrained air, the void fraction and the evolution of the bubble size distribution together with the dependence of these quantities on the turbulent dissipation rate and the initial wave slope. The results are based on ensemble-averaged simulations, allowing a better resolution of the statistical properties of the bubbly flow.

The paper is organized as follows. Section 2 presents the numerical experiment and Section 3 the numerical results for both the time dependent and the time averaged variables of the problem. Section 4 presents an alternative phenomenological model, based on the idea that during the breaking and air entrainment process the mechanical dissipation scales with the work done against buoyancy forces. Our model is compatible with existing experimental results and the DNS results, both for the time-dependent and time-averaged bubble size distribution. Finally, this model is tested against available laboratory data and our numerical data and applied to scale the integral quantities of the flow in $\S 5$. Conclusions are presented in $\S 6$.

\section{Numerical experiments}

\subsection{The Gerris flow solver}

We solve the three-dimensional two-phase incompressible Navier Stokes equations accounting for surface tension and viscous effects using the open source solver Gerris (Popinet 2003, 2009), based on a quad/octree adaptive spatial discretization, multilevel Poisson solver. The interface between the high density liquid (water) and the low density gas (air) is reconstructed by a geometric Volume Of Fluid (VOF) method. The multifluid interface is traced by a function $\mathcal{T}(\mathbf{x}, t)$, defined as the volume fraction of a given fluid in each cell of the computational mesh. The density and viscosity can thus be written as $\rho(\mathcal{T})=T \rho_{w}+(1-\mathcal{T}) \rho_{a}, \mu(\mathcal{T})=\mathcal{T} \mu_{w}+(1-\mathcal{T}) \mu_{a}$, with $\rho_{w}, \rho_{a}$ and $\mu_{w}, \mu_{a}$ the density and viscosity of the two fluids (water and air), respectively. The incompressible, variable density, Navier-Stokes equations with surface tension can be written as

$$
\begin{gathered}
\rho\left(\partial_{t} \mathbf{u}+(\mathbf{u} \cdot \nabla) \mathbf{u}\right)=-\nabla p+\nabla \cdot(2 \mu \mathbf{D})+\gamma \kappa \delta_{s} \mathbf{n} \\
\partial_{t} \rho+\nabla \cdot(\rho \mathbf{u})=0 \\
\nabla \cdot \mathbf{u}=0
\end{gathered}
$$

with $\mathbf{u}=(u, v, w)$ the fluid velocity, $\rho \equiv \rho(\mathbf{x}, t)$ the fluid density, $\mu \equiv \mu(\mathbf{x}, t)$ the dynamic viscosity and $\mathbf{D}$ the deformation tensor defined as $D_{i j} \equiv\left(\partial_{i} u_{j}+\partial_{j} u_{i}\right) / 2$. The Dirac delta, $\delta_{s}$, expresses the fact that the surface tension term is concentrated on the interface, where $\gamma$ is the surface tension coefficient, $\kappa$ and $\mathbf{n}$ the curvature and normal to the interface.

This solver has been successfully used in multiphase problems like atomization (Fuster et al. 2009; Agbaglah et al. 2011; Chen et al. 2013), the growth of instabilities at the interface (Fuster et al. 2013), wave breaking in two (Deike et al. 2015) and three dimensions (Fuster et al. 2009), capillary wave turbulence (Deike et al. 2014) and splashing (Thoraval et al. 2012).

\subsection{Interface reconstruction and bubble counting}

In Gerris, the interface between volumes of water $(\operatorname{tracer} \mathcal{T}=1)$ and air $(\operatorname{tracer} \mathcal{T}=0)$ is reconstructed by a discrete geometric VOF method (Scardovelli \& Zaleski 1999). In the

geometric Volume of Fluid formulation, the volume fraction field is the exact integral of 


\begin{tabular}{|c|c|c|c|c|}
\hline Set & $\mathrm{S}$ & Resolution & $\begin{array}{l}\text { Ensemble } \\
\text { size }\end{array}$ & Figure \\
\hline A: Various slopes & 0.36 & $512^{3}$ & 1 & Fig. 5b; 14 \\
\hline- & 0.40 & $512^{3}$ & 1 & Fig. 5b; 14 \\
\hline- & 0.42 & $512^{3}$ & 1 & Fig. 5 b; $6 ; 14$ \\
\hline- & 0.45 & $512^{3}$ & 1 & Fig. $5 b ; 14$ \\
\hline- & 0.47 & $512^{3}$ & 1 & Fig. 5b; 14 \\
\hline- & 0.49 & $512^{3}$ & 1 & Fig. 5b; 14 \\
\hline- & 0.51 & $512^{3}$ & 1 & Fig. 5b; 14 \\
\hline- & 0.53 & $512^{3}$ & 1 & Fig. 5b; 14 \\
\hline- & 0.55 & $512^{3}$ & 1 & Fig. $2 ; 5 \mathrm{a}, \mathrm{b} ; 6 ; 14$ \\
\hline- & 0.57 & $512^{3}$ & 1 & Fig. 5b; 14 \\
\hline- & 0.6 & $512^{3}$ & 1 & Fig. 5b; 14 \\
\hline- & 0.63 & $512^{3}$ & 1 & Fig. 5b; 14 \\
\hline B: Mesh convergence study & 0.55 & $256^{3}$ & 1 & Fig. $15 ; 16$ \\
\hline- & 0.55 & $512^{3}$ & 1 & Fig. $15 ; 16$ \\
\hline- & 0.55 & $1024^{3}$ & 1 & Fig. $3 ; 4 ; 15 ; 16$ \\
\hline C: Ensemble average & 0.42 & $512^{3}$ & 10 & Fig. 9; 11d; 12; 1314 \\
\hline- & 0.47 & $512^{3}$ & 6 & Fig. $9 ; 11 d ; 12 ; 13 ; 14$ \\
\hline- & 0.51 & $512^{3}$ & 6 & Fig. $8 \mathrm{~b} ; 9 ; 11 \mathrm{~d} ; 12 ; 13 ; 14$ \\
\hline- & 0.55 & $512^{3}$ & 6 & Fig. $7 ; 8 ; 9 ; 11 ; 12 ; 13 ; 14$ \\
\hline- & 0.6 & $512^{3}$ & 6 & Fig. $8 \mathrm{~b} ; 9 ; 11 \mathrm{~d} ; 12 ; 13 ; 14 ; 17$ \\
\hline
\end{tabular}

Table 1: Parameters of the three sets of DNS of three-dimensional breaking waves, $B o=$ $200, R e=40000$. The last column indicates in which figures of the paper the data are used.
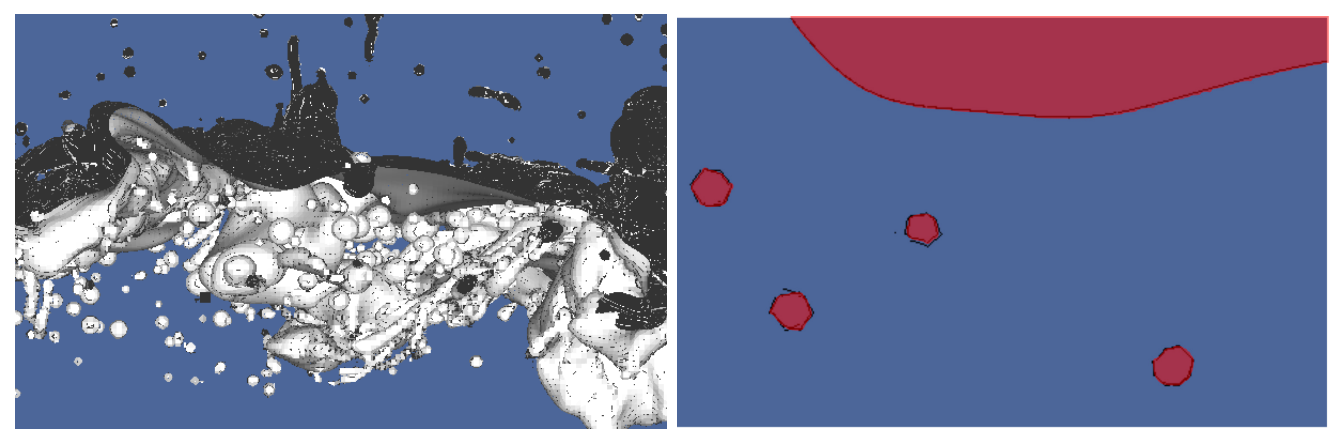

Figure 2: Left: example of the VOF interface after wave breaking, with numerous visible bubbles in water and droplets in the air. Right: Zoom and cut in the $y=0$ plane of the left figure, showing the principle of the bubble (and droplet) counting method and volume measure. Each closed surface of air in the plane is shown in red, corresponding to $\mathcal{T}=0$ with 4 bubbles and the main ambient gas area on top of the water. The area in blue corresponds to the water, with $\mathcal{T}=1$. 
the volume fraction in each discretisation element. It is not "smeared" or "diffused", i.e. the volume fraction is one or zero in cells which do not contain an interface and between zero and one in cells which contain an interface. The interface can then be reconstructed unambiguously in each cell with second-order accuracy (using piecewise-linear elements). These reconstructed piecewise-linear elements are displayed graphically in Figures 2, 3 and 4 . These images are thus directly representative of the accuracy of the interface representation by the VOF method.

The volume of individual bubbles and droplets can then be determined without ambiguity by considering connected regions, separated by interfacial cells. This is done in practice by using an implementation of the classical "painter's algorithm" which is typically used in bitmap graphics editors to "fill" connected regions of an image with a given color. This principle is illustrated in Figure 2, showing a full 3D image of bubbles under a breaking wave together with a $2 \mathrm{D}$ cut in the transverse $y$ plane.

The volume of air (water) is then defined by the sum over all closed surfaces corresponding to $\mathcal{T}=0$ of volume $v_{i}^{a}\left(\mathcal{T}=1\right.$, of volume $\left.v_{i}^{w}\right)$. Denoting the main volume of air above the water $v_{0}^{a}$ (initially half of the numerical domain), the volume of air in the water, i.e. the volume of air entrained by a breaking wave in our case, is then $V=\sum_{i \geqslant 1} v_{i}^{a}$. Symmetrically, the volume of ejected water is $V_{w}=\sum_{i \geqslant 1} v_{i}^{w}$. This method is exact at the order of the resolution of the Navier Stokes equations and the associated VOF method; each closed surface being detected and counted without ambiguity.

Volume and mass conservation during the breaking process can be investigated by computing the total amount of air (water) in the simulation, $V_{a}^{\text {total }}=\sum_{i \geqslant 0} v_{i}^{a}\left(V_{w}^{\text {total }}=\right.$ $\sum_{i \geqslant 0} v_{w}$ ). As shown in Appendix A, errors in mass (or volume) conservation are very small in the present numerical methods (Popinet 2003, 2009). We have checked here that, mass is conserved to better than $0.01 \%$ for both air and water for all resolutions tested and better than $0.001 \%$ in the highest resolution case (equivalent to $1024^{3}$, see Appendix A).

\subsection{Initial conditions and physical parameters}

We study a single breaking wave as was done in a previous 2D study (Deike et al. 2015), but now extending it to three-dimensions.

A third-order Stokes wave solution for the interface $\eta(x, y)$ and the velocity potential $\phi(x, y, z)$ in the water are used as initial conditions in a square box of size $\lambda$ on a side (see Deike et al. (2015)). The wave propagates in the $x$ direction. Boundary conditions are periodic in $x$ and $y$, and the top and bottom walls are free-slip (at $z= \pm H=\lambda / 2$ ). The wave slope $S=a k$, with $a$ the initial wave amplitude and $k=2 \pi / \lambda$ the wavenumber, varies from 0.35 to 0.65 , i.e. from incipient wave breaking to strongly plunging waves (Deike et al. 2015). Note that since we are using only the third-order Stokes wave solution, slopes higher than the limiting slope for the full Stokes wave solution can be defined.

The density and viscosity ratios of the two phases are those of air and water. The Bond number $B o=\Delta \rho g /\left(\gamma k^{2}\right)$, with $\Delta \rho$ the density difference between the two fluids, $g$ the gravity and $\gamma$ the surface tension, gives the ratio between gravity and surface tension forces. Due to computer limitations, related to the range of scales we are able to resolve, we choose $B o=200$. That corresponds to $\lambda=24 \mathrm{~cm}$ in air-water conditions; large enough to generate a plunging breaker while also including the surface tension effect, necessary to correctly resolve bubbles and droplets. The Reynolds number in the liquid is defined by $R e=c \lambda / \nu$, with $c=\sqrt{g / k}$ the linear deep-water gravity-wave phase speed and $\nu$ the kinematic viscosity of the liquid (water). Again, due to computer limitations, related to spatial resolution constraints, we use $R e=40000$, to correctly resolve the viscous 
boundary layer and still be at a large Reynolds number. This value is smaller than the one for a real $24 \mathrm{~cm}$ wave by about a factor of 3 , but should not affect qualitatively the results since we are at a sufficiently high Reynolds number (Deike et al. 2015).

Adaptive mesh refinement is used to accurately solve for the interface and the vortical structures, with an equivalent grid resolution of $512^{3}$ for most of the runs, leading to a mesh size $\lambda / 512 \approx 0.4 \mathrm{~mm}$ on the interface. This configuration allows accurate solutions for the dissipative scales, as shown by previous two-dimensional simulations (Deike et al. 2015) and all the relevant length scales of the bubble formation problem: the wave scale, the capillary length $\left(l_{g c}=\sqrt{\gamma /(\Delta \rho g)}\right)$ and the Hinze scale.

The energy components of the propagating wave can easily be obtained by integration over the whole volume, $\mathcal{V}$, of air and water and are respectively the kinetic energy, $E_{k}=\frac{1}{2} \int_{\mathcal{V}} \rho \mathbf{u}^{2} d \mathcal{V}$, the gravitational potential energy: $E_{g}=\int_{\mathcal{V}} \rho g z d \mathcal{V}+\lambda / 8$, the constant $\lambda / 8$ coming from the fact that the bottom of the box, $z=-\lambda / 2$, is used as a vertical datum (Chen et al. 1999), and the surface tension potential energy: $E_{s}=\gamma\left(\mathcal{L}-\lambda^{2}\right)$, where $\mathcal{L}$ is the surface area of the interfaces including those of the bubbles. The total energy is then $E=E_{k}+E_{g}+E_{s}$. In the following, these quantities will be given per unit length of breaking crest, i.e. divided by the width of the simulation box $\lambda$. Note also that the length of breaking crest $L_{c}$ is the width of the simulation box, $L_{c}=\lambda$.

\subsection{Summary of the runs}

Three sets of numerical experiments are presented and summarized in table 1 . The set A of experiments consists of 12 runs for waves with various initial slopes (from $S=0.35$ to $S=0.65$ ), with no initial perturbation in the transverse $y$ direction. The set B of experiments is a mesh size dependence study and is described in Appendix A. Three runs are performed with increasing resolutions (and same initial slope, $S=0.55$, corresponding to a plunging breaker) and show that the result are not changed for resolution finer than the grid equivalent to $512^{3}$. Finally, the third set of experiments consists of ensemble averages for 5 different initial slopes $S$ in the same range as before. For each slope $S$, between 6 and 10 runs are performed with some initial perturbation in the transverse direction $y$. An example of the obtained data set and the ensemble-averaged results is given in Appendix B. It shows good statistical convergence and that small perturbation do not significantly change the wave dissipation and bubble dynamics; however the ensemble averaging improves the statistical convergence of the bubble size distribution. Table 1 also shows in which figure the various runs are used.

\section{Wave breaking dynamics, air entrainment and energy dissipation}

\subsection{Wave breaking}

Figure 3 shows the interface $\eta(x, y, t)$ evolution with time for a plunging breaker of initial slope $S=0.55$. The dynamics of a plunging breaker have been described by several authors. A jet forms in front of the wave $(\mathrm{a}, \mathrm{b})$, strikes the surface (c), and falls back to the surface due to gravity. Before the jet reconnects, the wave dynamics remain mostly two-dimensional. Air entrainment then occurs through different mechanisms, recently summarized in the review by Kiger \& Duncan (2012): the entrapment of an air pocket when the jet reconnects the water, entraining a large cavity and large bubbles; entrainment around the jet impact site entraining smaller bubbles; entrapment by the subsequent splashes events; entrainment by the turbulent breakdown of the forward face of the wave. Indeed, numerous bubbles and droplets are visible $(\mathrm{c}, \mathrm{d}, \mathrm{e})$ while the flow has become fully three-dimensional. Entrainment of air also occurs when the jet impacts the surface (c), during the subsequent splashing $(\mathrm{d}, \mathrm{e})$, and when high velocity droplets fall back into the 

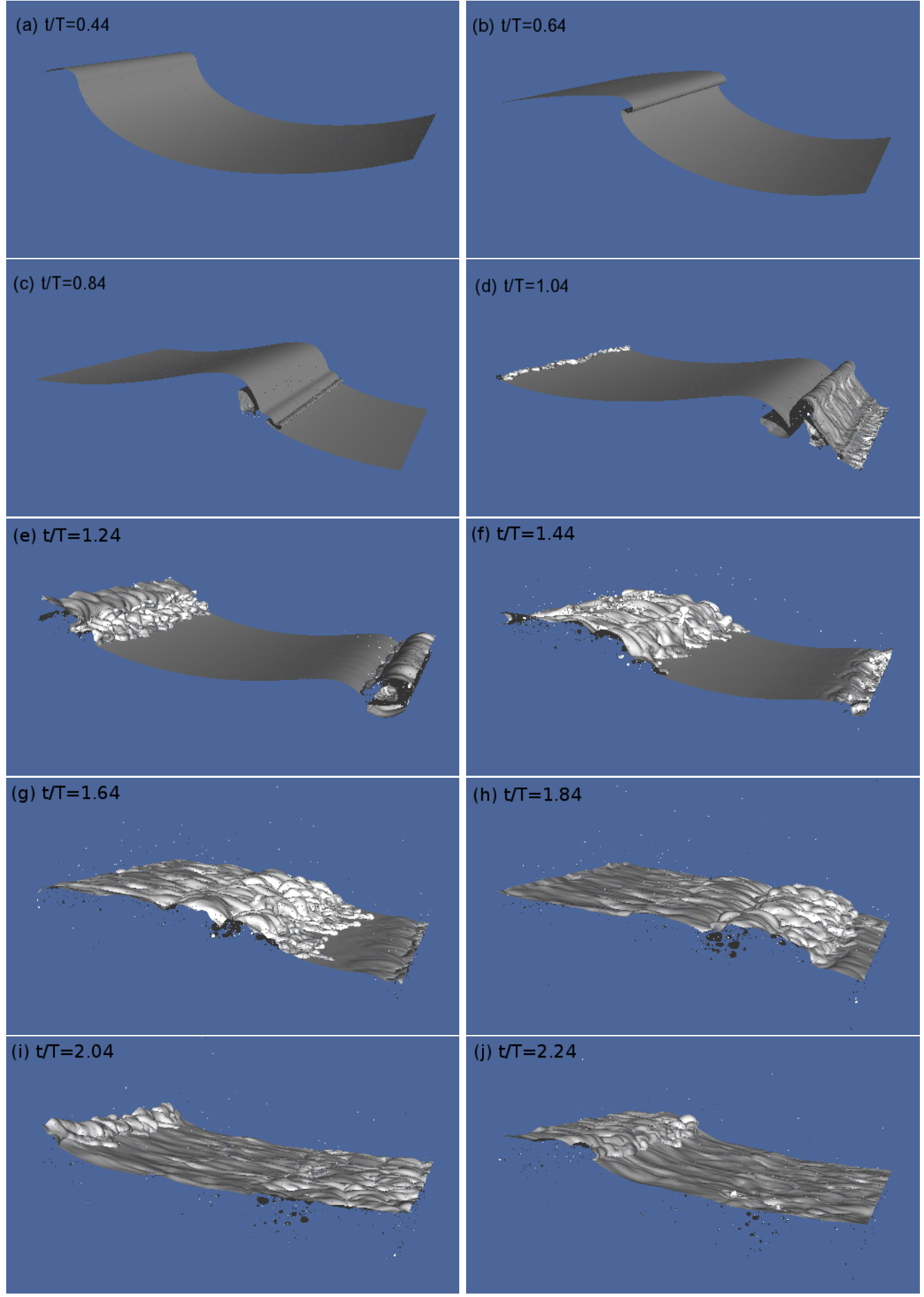

Figure 3: Snapshots of 3D Gerris DNS solutions for the interface, at different times, for a plunging breaker, $S=0.55$ and a $1024^{3}$ equivalent resolution. (a, b) formation of the jet; (c) impact and entrainment of the initial air pocket; (d, e) splashing and fragmentation of the air pocket; (f, g) splashing and bursting of large bubbles; (h to j) large dense bubble plume with numerous bubbles of various sizes in the water, together with droplets in the air. 
water (f,g). As discussed by Deane \& Stokes (2002); Kiger \& Duncan (2012), there are two main steps in bubble formation, first when the jet impact the water surface, creating relatively small bubbles and second when the air cavity collapses, creating larger bubbles that are then fragmented by turbulent fluctuations. Up to a thousand bubbles are counted in this simulation $(f, g)$. The bubble cloud is very dense just after impact (f,g,h), and then bubbles rise to the surface and burst, with the larger bubbles rising faster (i to 1). The waves then continue to propagate leaving the bubble cloud behind.

Figure 4 shows a view from bottom of the same simulation, revealing insights on the entrainment and bubble break-up process. After the wave impact, we can see the large air cavity entrained in the water, together with some satelite bubbles of small size (a). Just after the initial entrainment of the cavity, it is connected to the main ambient gas phase by thin filaments of air (b). These filaments of air are commonly observed under breaking waves in surf movies and were recently discussed in detail by Lubin \& Glockner (2015). Later on, the cavity starts to collapse, creating both large and small bubbles $(\mathrm{c}, \mathrm{d})$. Once the cavity is completely destroyed, we observe a dense bubble plume, with bubble radii varying over more than one order of magnitude.

The evolution of the wave is similar for the various plunging breakers $S \gtrsim 0.42$, with increasing slopes leading to an increase of the entrained air. For smaller slopes, a spilling process is observed $(0.35 \lesssim S \lesssim 0.42)$, with a dynamic similar to the one described experimentally by Rojas \& Loewen (2010).

\subsection{Energy dissipation}

The first step is to verify that we correctly capture the wave dissipation properties in the present three-dimensional work. Wave energy dissipation due to breaking can be written as $E_{d i s s}=\int \epsilon_{l} \Lambda(\mathbf{c}) d \mathbf{c}$, with $\epsilon_{l}$ the dissipation per unit length of breaking crest and $\Lambda(\mathbf{c}) d \mathbf{c}$ the mean length of breaking wave fronts moving at phase velocities in the range $(\mathbf{c}, \mathbf{c}+d \mathbf{c})$ (Phillips 1985). The breaking distribution $\Lambda(\mathbf{c})$ can be measured in the field (Melville \& Matusov 2002; Gemmrich et al. 2008; Thomson et al. 2009; Kleiss \& Melville 2010; Sutherland \& Melville 2013), while the dissipation $\epsilon_{l}$ is related to the fluid properties and the breaking kinematics (Duncan 1981; Phillips 1985),

$$
\epsilon_{l}=b \rho c^{5} / g
$$

where $g$ is gravity, $\rho$ the water density, $c$ the characteristic phase speed of the breaking front, and $b$ the non-dimensional breaking parameter.

The local time-averaged dissipation rate $\bar{\varepsilon}$ is related to the dissipation rate per unit breaking crest length, $\epsilon_{l}$, by (Drazen et al. 2008)

$$
\epsilon_{l}=\rho A \bar{\varepsilon},
$$

with $A=\pi h^{2} / 4$ representing the cross-sectional area of the entrained cylinder of air. This assumes that the turbulence is homogenous is the cylinder of cross-sectional area $A$ created by the breaking event (Drazen et al. 2008). The relationship between $\bar{\varepsilon}$ and the wave height at breaking $h$ is given by the inertial scaling for breaking waves (Drazen et al. 2008): $\bar{\varepsilon}=\Xi h^{1 / 2}(2 g)^{3 / 2}$, with $\Xi$ an $\mathrm{O}(1)$ constant. This leads to $\epsilon_{l}=\Xi \rho g^{3 / 2} h^{5 / 2} \pi / \sqrt{2}$, which combined with Eq. 3.1 gives

$$
b=\Xi \frac{\pi}{\sqrt{2}}(h k)^{5 / 2},
$$

where $h k$ is a measure of the wave slope at breaking. Drazen et al. (2008) have shown that the slope at breaking $h k$ is approximatively proportional to the linearly predicted maximum slope, $S$, of a focusing packet. Romero et al. (2012) derived the following 

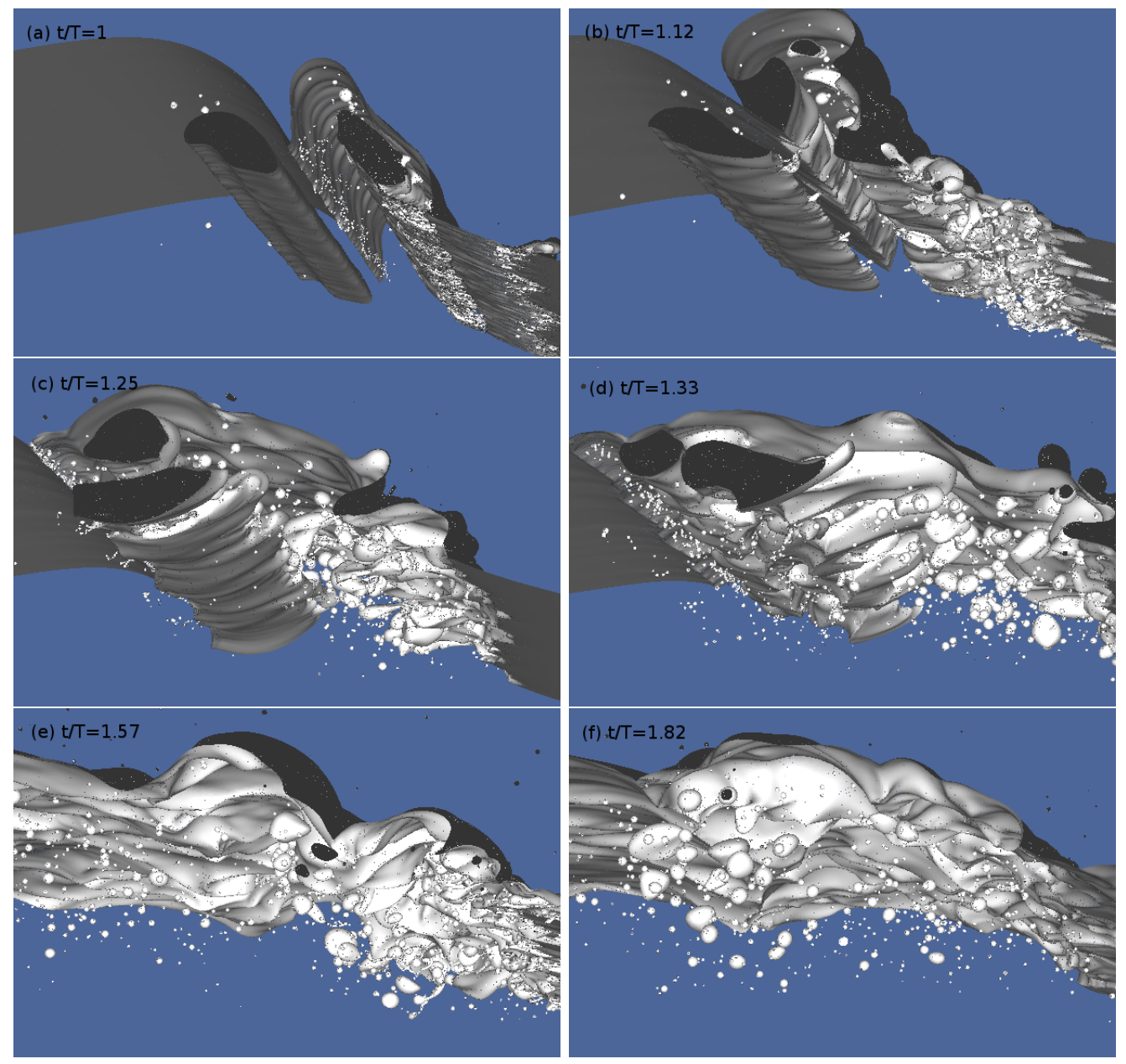

Figure 4: Bottom view of the simulation, showing the entrainment and collapse of the air cavity during the breaking event. (a) Just after impact, the tube of air is already formed inside the water, with some small satelite bubbles nearby. (b) The air cavity has started to collapse and is connected to the main surface by thin filaments of air. Bubbles of various sizes start to be visible. In (c) and (d) the cavity continues to collapse, with numerous bubbles of various sizes. In (e) and (f) the cavity has completely collapsed, and a dense bubble plume is visible, with bubbles with radii varying over one order of magnitude.

semi-empirical relation for the breaking parameter initially based on laboratory data,

$$
b=0.4(S-0.08)^{5 / 2},
$$

where $S_{0}=0.08$ is a measure of the threshold for breaking and 0.4 an evaluation of the constant $\Xi \pi / 2$. This inertial model, based on a simple physical argument for strong plunging waves, has been confirmed through extensive experimental studies and modeling, well beyond the region of validity of the initial hypothesis (Romero et al. 2012; Grare et al. 2013; Pizzo \& Melville 2013; Melville \& Fedorov 2015; Deike et al. 2015). Note that proportionality between the initial slope and the slope at breaking is also true in our DNS of steep Stokes waves, and following the definition of Drazen et al. (2008) where $h$ 

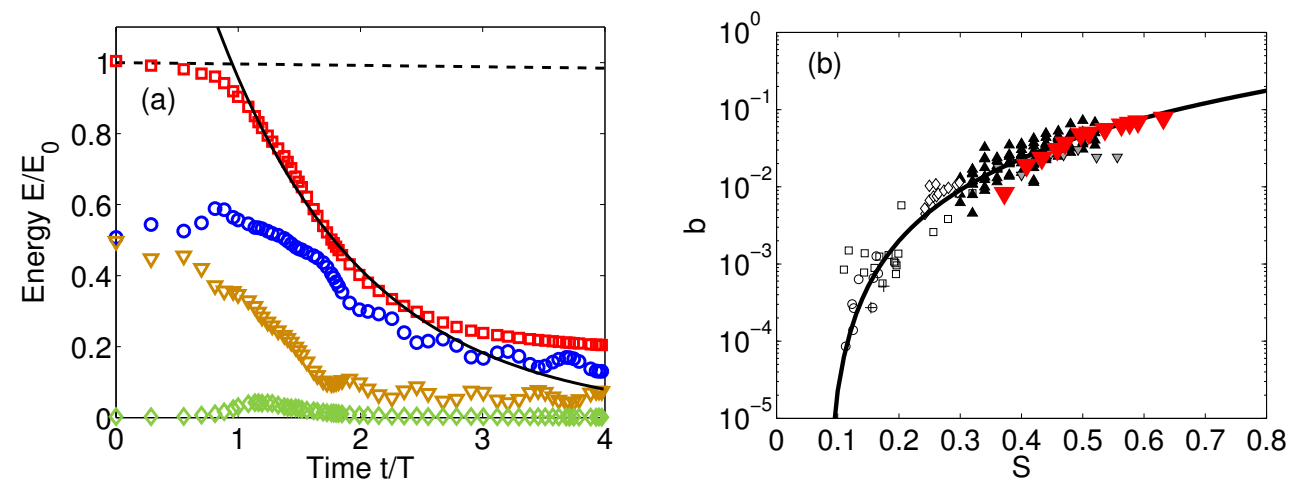

Figure 5: (a) Wave energy as a function of time. ( $\square$ ): total energy, (०): kinetic energy, $(\nabla)$ gravitational potential energy, $(\diamond)$ : surface tension potential energy. The solid line is an exponential fit $E=E_{0} e^{-\zeta t}$ for $1<t / T<2$, and the dashed line is the linear viscous decay $E=E_{0} e^{-2 \nu k^{2} t}$. (b) Breaking parameter $b$ as a function of the initial wave slope $S$. ( $\mathbf{\nabla})$ : DNS data. Solid line: semi-empirical formulation based on scaling argument, Eq. $3.4, b=0.4(S-0.08)^{5 / 2}$ Romero et al. (2012). Black and grey symbols are experimental data; black and grey triangles and grey diamonds are from Drazen et al. (2008), cross and circle are from Banner \& Peirson (2007) and squares are from Grare et al. (2013). The differences between experiments and DNS at lower values of $S$ come from differences in initiating wave breaking.

is the vertical distance the breaking wave toe travels before impact, $h k=-0.05+S$ for both the 3D DNS presented here and the 2D results presented in Deike et al. (2015). In the following we now consider $S$ as our initial wave slope.

Figure 5a shows the evolution with time of the total wave energy $E$ per unit length of breaking crest, together with the kinetic and potential components. An abrupt decrease of the total wave energy is observed when the wave breaks with most of the wave energy lost during the first wave period. The energy decay can be fitted by an exponential during this time period, $E=E_{0} \exp (-\zeta t)$, with $\zeta$ the observed decay rate and $E_{0}$ the initial wave energy per unit length of crest. The dissipation rate per unit breaking crest is then simply given by $\epsilon_{l}=E_{0} \zeta$ (or by taking $\epsilon_{l}=-\Delta E / \tau_{b}$, where $\tau_{b}$ is the active breaking time and $\Delta E$ the energy difference before and after breaking, both estimations being equivalent). The breaking parameter is then $b=\epsilon_{l} g /\left(\rho c^{5}\right)$, with $c$ the linear phase speed of the wave.

Figure 5b shows $b$ as a function of the initial wave slope $S$ in the DNS, and we observe a very good agreement for strong plunging waves with the semi-empirical result given by Eq. 3.4 initially derived from laboratory data (Drazen et al. 2008; Romero et al. 2012), see also data from Grare et al. (2013) and two-dimensional numerical simulations from Deike et al. (2015). This confirms that the present three-dimensional DNS captures the dissipative scales of the breaking wave process. The total dissipation due to breaking in the three-dimensional DNS is very similar to the one obtained in two-dimensional DNS reported by Deike et al. (2015). The difference in the dissipation between experiments and DNS for $S$ between 0.35 and 0.4 is most likely related to the route to breaking, i.e. how the initial data are set up. Indeed, as discussed in Deike et al. (2015), the critical slope for wave breaking changes when a steep Stokes wave is used instead of wave focusing packet or modulational instability in the laboratory. In the present case, a slope of 0.35 
corresponds to an incipient breaking wave, which has total dissipation less than a spilling breaker of the same slope obtained by a wave focusing technique in the laboratory.

\subsection{Air entrainment and void fraction}

Experimentally, the local void fraction $\alpha(x, z, t)$ (volume of air per unit volume of water) is measured using conductivity (Lamarre \& Melville 1991) or optical probes (Blenkinsopp \& Chaplin 2007; Rojas \& Loewen 2007). A spatial map of the void fraction is then obtained by repeating this measure at various locations. In order to compare our simulations with the experimental description of the two-phase air water flow during the breaking event, we compute an equivalent of this experimentally measured void fraction, by averaging the tracer $\mathcal{T}$ over the transverse direction $y$ :

$$
\alpha(x, z, t)=\frac{1}{L_{c}} \int_{-L_{c} / 2}^{L_{c} / 2} \mathcal{T}(x, y, z, t) d y,
$$

where $L_{c}=\lambda$ is the size of the domain in the transverse $y$ direction (and corresponds to the length of breaking crest). This measure of the void fraction can then be integrated in space in order to obtain another estimate the amount of air entrained by the breaking wave. The main interface during the breaking process can then be defined by the $\alpha(x, z, t)=50 \%$ value, as proposed experimentally by several authors (Lamarre \& Melville 1991; Blenkinsopp \& Chaplin 2007).

Figure 6 shows the evolution of the void fraction (volume of air per unit volume) $\alpha(x, z, t)$ during the breaking process, for a plunging breaker $(S=0.55)$ and a spilling breaker $(S=0.43)$.

In the plunging case, the impact of the jet reveals again the various air entrainment processes, the entrapment of a large air pocket (a-b), as well as the rebound (b-c) and entrainment by the turbulent breakdown of the forward face. Spray generation is observed during the splashing process (c-d). Bubble plumes are formed during these entrainement processes and high void fraction areas are visible (c-f). The bubble plume appears very dense and consists predominantly of large air cavities, that collapse into smaller bubbles and are driven down into the water reaching a maximum penetration depth (d-f). Then the bubble clouds spread horizontally and large bubbles and air cavities rise back to the surface to burst, corresponding to the fast degassing stage (e-g). These events correspond to the active breaking stage. Smaller void fraction features remain for longer times (g-h), corresponding to the small bubbles with low rise velocities. Eventually, even the smaller bubbles rise back to the surface. This stage corresponds to the decay of the breaking event. The bubble cloud dynamics, as well as the void fraction observed during the breaking are consistent with previous experimental observations (Lamarre \& Melville 1991, 1994; Blenkinsopp \& Chaplin 2007): the air cavity is first entrained with $\alpha=100 \%$, collapses and gives birth to a bubble cloud with $\alpha$ up to $30 \%$ during the active breaking stages.

In the spilling case, the breaking process is started by the appearance of a rough surface or of a small jet at the wave crest (i), then a small region of turbulence forms at the crest of the wave, and this region grows as water spills down the face of the wave $(\mathrm{j}, \mathrm{k})$. As the wave spills, air is entrained and spray is ejected at the leading edge of the breaker $(\mathrm{k}, \mathrm{l})$ and relatively shallow bubble clouds are formed beneath the surface (l-o). This general scenario is in agreement with experimental observations from Rojas \& Loewen (2010). As for the plunging case, larger bubbles rise back to the surface and burst while smaller ones remain longer in the water column (m-p). 


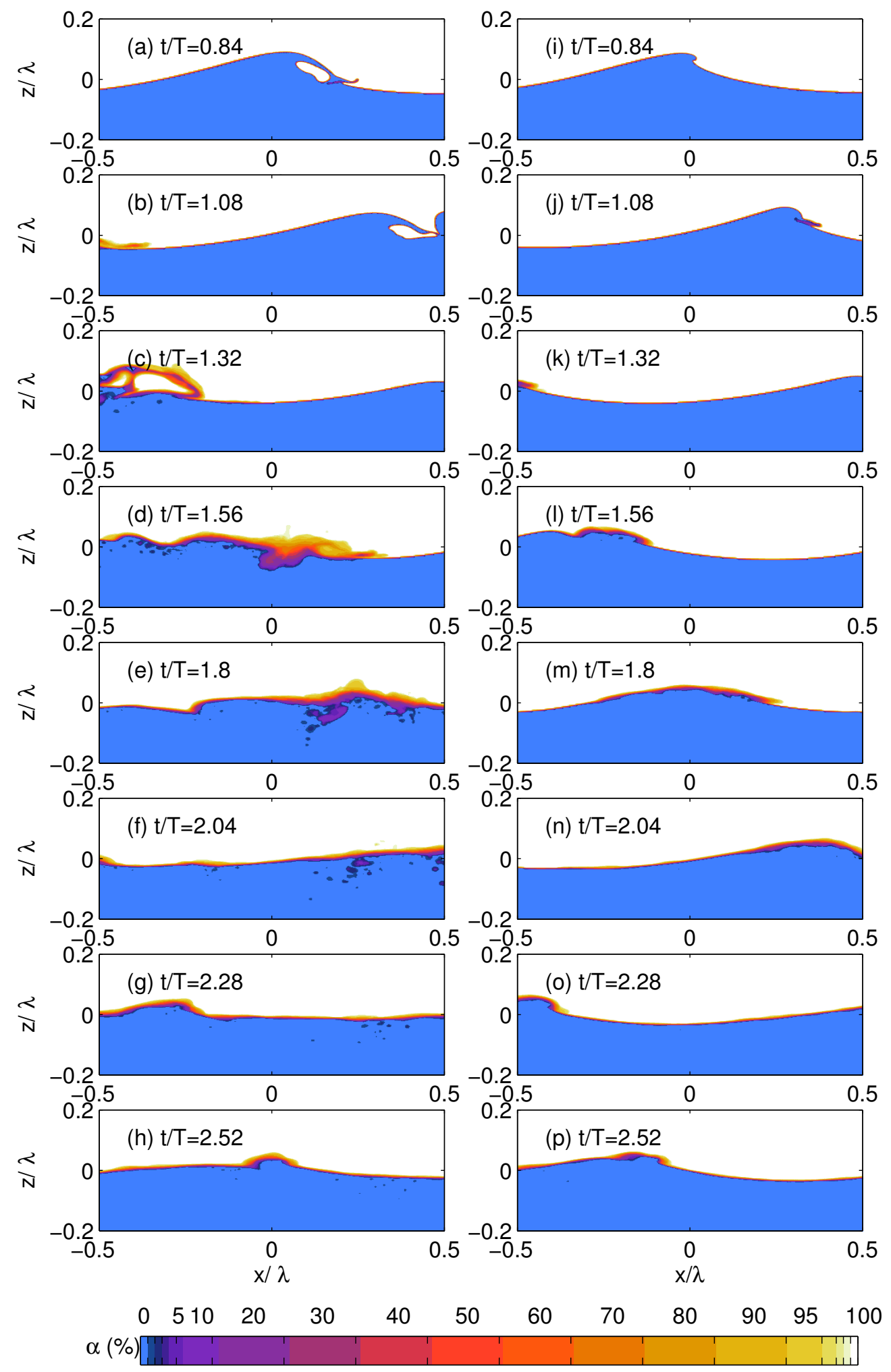

Figure 6: Transversally-integrated void fraction $\alpha(x, z, t)=\frac{1}{L_{c}} \int_{-L_{c} / 2}^{L_{c} / 2} \mathcal{T}(x, y, z, t) d y$, (in $\%$ ), from the 3D data, at different times. Left: plunging breaker (panels a-h, $S=0.55$ ). Right: spilling breaker (panels i-p, $S=0.42$ ). Color scale is non-linear in order to reveal variations towards the extremes of the range. 

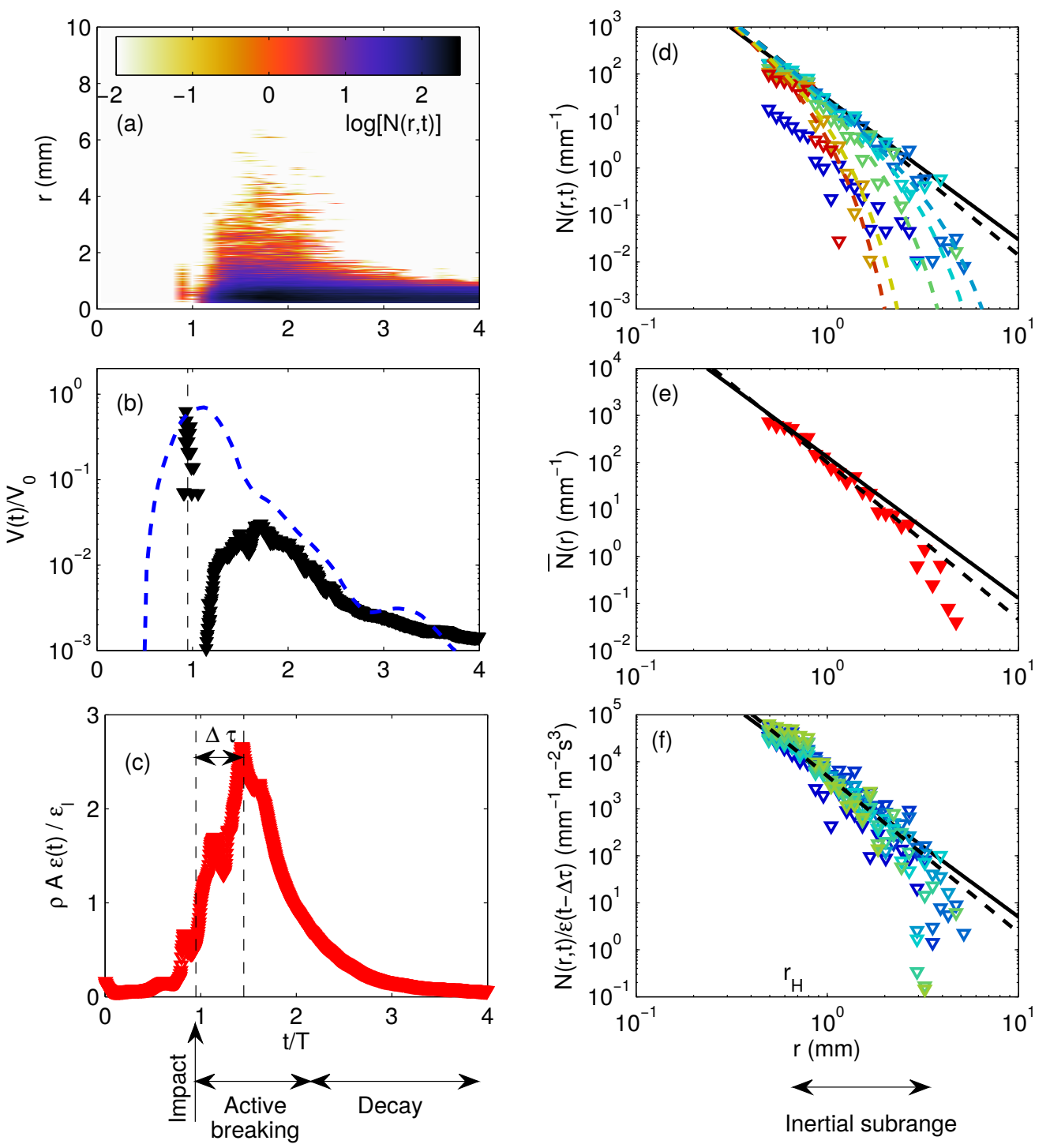

Figure 7: Ensemble-averaged data for a plunging breaker $(S=0.55)$ (a) Time evolution of the bubble size distribution $N(r, t)$. Color is in log-scale. (b) Time evolution of the volume of entrained air $V(t) / V_{0}$, with $V_{0}=A L_{c}$, calculated from the closed surfaces $(\boldsymbol{\nabla})$ and from the void fraction (blue line). (c) Time evolution of the energy dissipation $\rho A \varepsilon(t) / \epsilon_{l}$ (red symbols). A delay between the time $\varepsilon(t)$ and $V(t)$ maxima is clearly visible. (d) Bubble size distribution $N(r, t)$ at different times $t$. Time is color coded. Dashed lines are $N(r, t) \propto r^{-m} e^{\left[-K\left(r / r_{m}(t)\right)^{2}\right]}$, with $K=2$, and $r_{m}(t)$ decreasing with time. (e) Active breaking time-averaged bubble size distribution $\bar{N}(r)$ : the dashed line is $\bar{N}(r) \propto r^{-10 / 3}$ and the solid line is $\bar{N}(r) \propto r^{-3}$. (f) Rescaled bubble size distribution $N(r, t) / \varepsilon(t-\Delta \tau)$ during the active breaking stage, from $t / T=1.2$ to $t / T=2.4$. $\Delta \tau$ is the delay time between the maximum entrained air and the maximum dissipation, corresponding to the turbulent break-up time. Time is color coded as in (d). 


\subsection{Time evolution of the bubble size distribution and integral quantities}

Here we describe the time evolution of the bubble plume properties (volume and size distribution) for the strong plunging wave, $S=0.55$ shown in Figs. 3, 4, 5a and 6 . The data are obtained from ensemble averaging 6 runs with small initial perturbation in the transverse wave direction $y$. As shown in Appendix B, the ensemble averaging improves the bubble statistics.

Figure 7a shows the time evolution of the bubble size distribution $N(r, t)$. When the jet reconnects to the water surface $(t / T \approx 1)$, a large pocket of air is entrained in the water. This pocket of air collapses and bubbles of various sizes are formed. Bubbles between 2 $\mathrm{mm}$ and the mesh size are first created at the impact $(t / T \approx 1)$, then a rapid growth of the bubble size distribution is observed $(1 \lesssim t / T \lesssim 1.6)$, with bubbles as large as 10 $\mathrm{mm}$, corresponding to the collapse of the air cavity. Therefore, bubbles of various sizes are created during the initial impact and entrapment of the air cavity (visible in Figure 4), due to entrainment by the jet, and at the edges of the air cavity, as discussed by Deane \& Stokes (2002) and Kiger \& Duncan (2012). The maximum number of bubbles in the system is reached at the end of this growing stage. A fast decay is then observed $(1.6 \lesssim t / T \lesssim 2.4)$. Large bubbles are the first to disappear, collapsing or rising back to the surface and bursting. These two stages define the active breaking time. Finally a slower decay of the size distribution is observed $(t / T>2.4$, decay stage) where small bubbles slowly rise to the surface.

Figure $7 \mathrm{~b}$ shows the evolution with time of the entrained volume of air $V(t) / V_{0}$, where $V_{0}=A L_{c}=\pi h^{2} L_{c} / 4$ is the reference maximum volume of air that can be entrained during the active breaking process. The volume of air is defined by the sum over all closed surfaces (bubbles of volume $v_{i}^{a}$ ), $V=\sum_{i \geqslant 1} v_{i}^{a}$, as described in $\S 2$. The first peak observed at $t / T \approx 1$ corresponds to the impact of the jet and the entrapment of a large pocket of air, with a cross-sectional area that is almost $\left(V=0.9 V_{0}\right)$ the reference tube of air of surface $A$, visible in Fig. 2, 4 and 5. The measured volume of air then decreases because of the collapse of the initial tube and its topological reconnection to the water surface during this highly turbulent event (as shown in Fig. 4b).

Then, bubbles are formed and a second maximum of the volume of air, which corresponds to the moment where the largest number of bubbles is present in the system. This second maximum is one order of magnitude smaller than the volume of the initial tube. The fraction of air that remains trapped in the water corresponds to the rapid and continuous increase of $V(t)$ for $1 \lesssim t / T \lesssim 1.6$. Then $V(t)$ decreases, first quickly over roughly one wave period $1.6 \lesssim t / T \lesssim 2.4$. As described by Lamarre \& Melville (1991), the fast decay is exponential, with $V=V_{0} \exp (-\kappa t / T)$, with $\kappa$ a numerical factor varying from approximately 2.5 to 4 in our DNS depending on the initial wave slope. These values appear in reasonable agreement with the experimental values, considering the difficulty of the experiments of Lamarre \& Melville (1991) and Blenkinsopp \& Chaplin (2007), who found $\kappa=3.9$ and $\kappa \approx 5$ respectively. This rapid decay corresponds to the fast degassing of the plume caused by large bubbles rising back to the surface. The growing stage and the fast decay correspond to the active breaking stages, lasting a time slightly larger than one wave period. It is followed by a slower decay, that was also observed experimentally by Lamarre \& Melville (1994).

Note that the abrupt decrease of air is related to the fact that the tube of air collapses and is partially reconnected to the air above the wave (as in Fig. 4b). If we follow the definitions used in laboratory experiments and compute the volume of entrained air based 
on the void fraction, (Lamarre \& Melville 1991; Blenkinsopp \& Chaplin 2007):

$$
V_{\alpha}(t)=L_{c} \int_{-L}^{L} d x \int_{-H}^{\alpha=50 \%} \alpha(x, z, t) d z,
$$

with $\alpha(x, z, t)=50 \%$ considered as the main wavy interface (and $L=\lambda$ the size of the numerical domain in the $x$ direction), then we observe a monotonic decay after the initial entrainment of the air cylinder at $t / T=1$ (as in the experiments). The two measures of the volume give the same result for the maximum volume of entrained air and for the time evolution after $t / T=1.6$.

Figure 7c shows the spatially averaged turbulent dissipation rate $\varepsilon(t)$ (normalized by $\epsilon_{l} /(\rho A)$ ) as a function of time $t$ (normalized by the wave period $T$ ). Its evolution follows closely the volume of entrained air $V(t)$, with a clear time delay $\Delta \tau$ between the maxima of $\varepsilon(t)$ and $V(t)$. The dissipation rate first increases during the air entrainment and splashing processes and then decreases during the degassing process. Note that during the active breaking stages, the instantaneous turbulent dissipation rate $\rho A \varepsilon(t)$ is of the same order of magnitude as the dissipation rate per unit length of breaking crest $\epsilon_{l}$ obtained from the decay of the wave energy (see Figure 5). When averaging in time, we obtain indeed $\rho A \bar{\varepsilon}=\epsilon_{l}$. The time lag between that in the volume of entrained air and the peak in the dissipation can be assigned to the fragmentation time of the initial air pocket.

Figure $7 \mathrm{~d}$ shows $N(r, t)$ at given times $t$ during the active breaking period and the decay stage. The time is color-coded. During this active breaking time $(1.2 \leqslant t / T \leqslant$ 2.4 ), the dependence on the radius is adiabatic in time, i.e the size distribution follows $N(r, t) \propto r^{-m}$, with $3 \leqslant m \leqslant 3.5$ constant with time, within the error bars.

The amplitude of the bubble size distribution first grows (from dark blue to light blue), reaches a maximum $(t / T \approx 1.9$, light blue), and then decays (from light green to red), following, with a time delay, the time evolution of both $\varepsilon(t)$ and $V(t)$. During this stage, the bubble cascade inertial subrange, i.e. the range of scales for which the power law is observed starts close to the smallest scale resolved in the simulation and ends around 4 $\mathrm{mm}$. The time evolution of $N(r, t)$ is in agreement with the experimental results from Blenkinsopp \& Chaplin (2010), where the bubble size distribution is found to follow a power law with $m \approx 3$ roughly constant during the active breaking stage.

After the active breaking stage $(t / T>2.4$, from yellow to red), the bubble size distribution rapidly decays, starting with the large bubbles and a much steeper bubble size distribution is observed, in agreement with experimental results (Terrill et al. 2001; Deane \& Stokes 2002). While Deane \& Stokes (2002) describe a steep power law (finding $m_{\text {decay }}=6$ ), the bubble size distribution can also be described by an empirical decay function; combining the turbulent breakup law $N(r, t) \propto r^{-m}$ and an exponential decay, due to the scale-dependent rise of the bubbles that governs the decay of $N(r, t)$. We propose the following empirical function:

$$
N(r, t)=N_{0} r^{-m} \exp \left[-K\left(\frac{r}{r_{m}(t)}\right)^{2}\right] .
$$

Here $r_{m}(t)$ is the time-dependent variable, $K=2$ a constant empirical parameter, and $m=10 / 3$. Here $r_{m}(t)$ corresponds physically to a cut-off radius that decreases with time, and quantifies the fact that, due to the rise of the bubbles, the inertial subrange of the bubble cascade is reduced once the active breaking stage is over. The exponent 2 inside the exponential functional form has been chosen to match the one giving the rise velocity of the bubbles (see below). For the times displayed in Fig. $7 \mathrm{e}, r_{m}(t)$ decreases linearly 
from $4 \mathrm{~mm}(t / T=2.5$, light blue) to $1 \mathrm{~mm}(t / T=3.5$, red $)$. Thus the cut-off radius decreases until it reaches a value close to the Hinze scale. At this time, the turbulent motion is too weak and no more bubble cascade process is observed. Note that the semiempirical formulation (Eq. 3.7) can be applied to the experimental data of Deane \& Stokes (2002) shown in the inset of Figure 1.

Figure 7e shows the time-averaged bubble size distribution $\bar{N}(r)$ over the active breaking time $\tau_{b} . \bar{N}(r)$ is found to follow a power law, $\bar{N}(r) \propto r^{-m}$, with $3 \leqslant m \leqslant 3.5$ compatible with various experimental results (Terrill et al. 2001; Deane \& Stokes 2002; Rojas \& Loewen 2007; Blenkinsopp \& Chaplin 2010). The bubble inertial subrange, i.e. the range of bubble radii for which the power law is observed, starts close to the smallest resolved scale (around $0.8 \mathrm{~mm}$ ) and ends at the radius of the largest bubbles observed in the simulations (between 4 and $6 \mathrm{~mm}$ ). The scale of the beginning of the bubble cascade corresponds to the Hinze scale $\left(r_{H} \approx 0.8 \mathrm{~mm}\right.$ using eq. 1.2). However, the mesh resolution is also close to this scale $(0.4 \mathrm{~mm})$ so the Hinze scale might not be fully resolved.

Note that, experimentaly, various shapes for $r<r_{H}$ have been observed by Blenkinsopp \& Chaplin (2010) Deane \& Stokes (2002) and Leifer \& de Leeuw (2006). We should also remark that it is clear from Figure $7 \mathrm{~d}$ that the exact value of the exponent $m$ depends on the time chosen to average the bubble size distribution. Indeed, if one averaged over the active breaking and decay stage, one would obtain a steeper size distribution and a higher value for $m$. We believe that this sensitivity in the time of observation is at least partially responsible for the relatively wide range of values observed for $m$ in the literature, due to the difficulty in properly identifying the active breaking time.

Finally, we discuss the scaling of $N(r, t)$ with $\varepsilon(t)$ during the active breaking stages. Figure $7 \mathrm{f}$ shows the size distribution is indeed well described by $N(r, t) \propto \varepsilon(t-\Delta \tau) r^{-m}$, with $3 \leqslant m \leqslant 3.5$ independent of time and $\Delta \tau$ the time lag related to the fragmentation of the initial air pocket. The time is color-coded as in Figure $7 \mathrm{~d}$. This description is valid, within the scatter of the data, for the active breaking stages that last more than one wave period $(1.2 \leqslant t / T \leqslant 2.4)$.

The time evolution of the entrained air phase can be used to define the active breaking stage; similarly to what was done experimentally using measurement of the noise generated by the bubbles (Drazen et al. 2008). The active breaking stage starts when the jet impacts the water surface and ends when the adiabatic bubble size distribution is no longer observed, so that its total duration here is $\tau_{b} \approx 1.2 T$.

The overall patterns of evolution of the volume of air, the dissipation rate, and the bubble size distribution, are similar for all plunging and spilling breakers we have investigated.

\subsection{Decay time and rising velocities}

The time evolution of the number of bubbles at a given scale $r$, is shown in Figure 8 normalized by its maximum $N(r, t) / N_{0}(r)$. Large bubbles are created first but the time delay between the maximum number of bubbles for various radii is small, indicating fast bubble break-up processes. Large bubbles are seen to disappear first and much faster than the smaller bubbles. The decay rate $\tau_{d}(r)$ of bubbles of radius $r$ can be measured by fitting the decay of the bubble size distribution by $N(r, t)=N_{0}(r) e^{-t / \tau_{d}(r)}$. This decay rate includes both the rise of the bubbles, and the collapse of the large bubbles into smaller ones. However, since we consider the decay starting at the end of the active breaking stage, the dominant process should be the rise of the bubble. From this decay rate, we can estimate an average bubble velocity by assuming that the path of the bubbles is given by the distance the bubble has to rise to reach the surface, which corresponds to the average penetration depth of the bubble cloud, say $h$. Thus we define the scale 

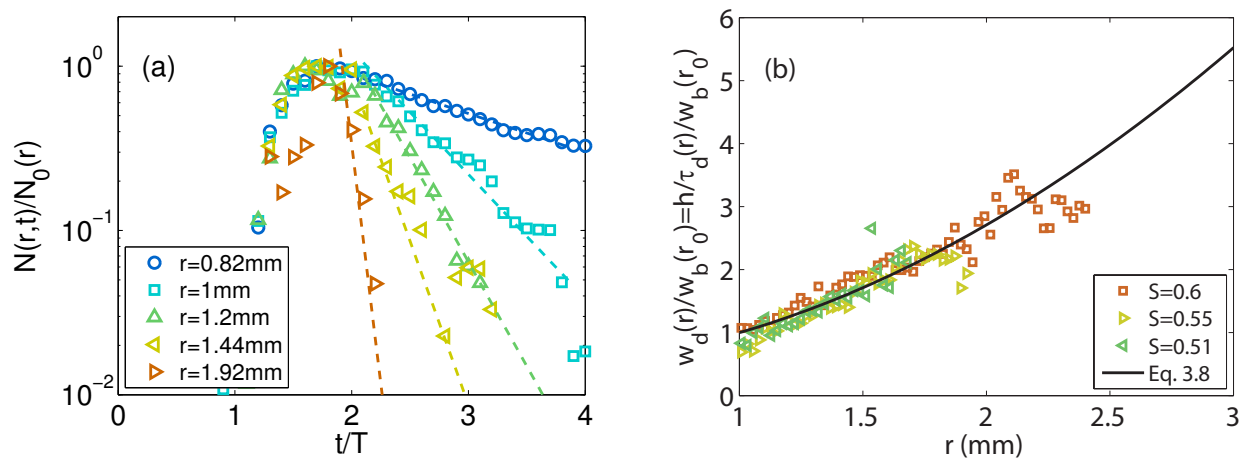

Figure 8: (a) Time evolution of $N(r, t)$ for $S=0.55$ and various radii $r$. Dashed lines are exponential fits, $N(r, t) / N_{0}(r)=e^{-t / \tau_{d}(r)}$. (b) Decay velocity $w_{d}(r)=h / \tau_{d}(r)$ compared to the rise velocity of bubbles $w_{b}$ (solid line) given by Eq. 3.8. Velocities are recaled by $w_{b}\left(r_{0}=1 \mathrm{~mm}\right)$.

dependent decay velocity of the bubble size distribution by $w_{d}(r)=h / \tau_{d}(r)$, with $w_{d}(r)$ shown in Figure $8 \mathrm{~b}$ for three plunging breakers.

The rise velocity of a bubble of radius $r$ in clean water (for radius larger than $100 \mu \mathrm{m}$ ) is given by Woolf \& Thorpe (1991) (see also Thorpe (1982) for a review of bubble rise velocities),

$$
w_{b}=\frac{g r^{2}}{\nu_{w}} \frac{1}{18\left[1-2 /(1+0.091 \chi)^{1 / 2}\right]}, \text { with } \chi=\frac{g r^{3}}{\nu_{w}^{2}} .
$$

Figure 8 shows that the decay velocity of the bubbles $w_{d}(r)=h / \tau_{d}(r)$ is close to the rise velocity of bubbles in clean water $w_{b}$ (Eq. 3.8). Thus the decay of the bubble plume (for $t / T>2$ ) is consistent with the bubbles rising back to the surface.

\subsection{Time-averaged bubble size distribution}

We now discuss the time-averaged (over the active breaking time $\tau_{b}$ ) bubble size distribution $\bar{N}(r)$ for various wave slopes $S$, and the relationship with the wave dissipation rate per unit length of breaking crest $\epsilon_{l}$.

Figure 9a shows the time-averaged bubble size distribution $\bar{N}(r)$, for increasing initial wave slope. The bubble size distribution follows a power law, $\bar{N}(r) \propto r^{-m}$ with $3 \leqslant m \leqslant$ 3.5 , from the Hinze scale to a cut-off radius $r_{m}$, that increases with $S$. The amplitude of $\bar{N}(r)$ as well as the range of bubble radii for which the power law is observed increase with the wave slope, $S$. Both are related to the increase of total volume of air entrained, the size of the initial air pocket trapped during the overturning and impact of the breaking wave, and the increase of turbulence fluctuations, with the slope, $S$. For a large amplitude plunging breaker, the bubble break-up cascade subrange goes from roughly $0.8 \mathrm{~mm}$ to $5 \mathrm{~mm}$ while for smaller amplitude spilling breakers, the range of validity of the $\bar{N}(r) \propto$ $r^{-m}$ relationship is smaller, the cut-off radius being around $3 \mathrm{~mm}$, closer to the Hinze scale, due to a smaller amount of entrained air and weaker turbulent fluctuations.

Figure $9 \mathrm{~b}$ shows the bubble size distribution rescaled by the dissipation rate per unit length of breaking crest $\epsilon_{l}, \bar{N}(r) / \epsilon_{l}$. A significant collapse of the data is observed within the bubble cascade subrange.

To summarize, we find that the dependence of the bubble size distribution on the bubble radius is the same for both the time-averaged data and the time-dependent data during the active breaking stage; both being proportional to $\propto r^{-m}, 3 \leqslant m \leqslant 3.5$ for a 

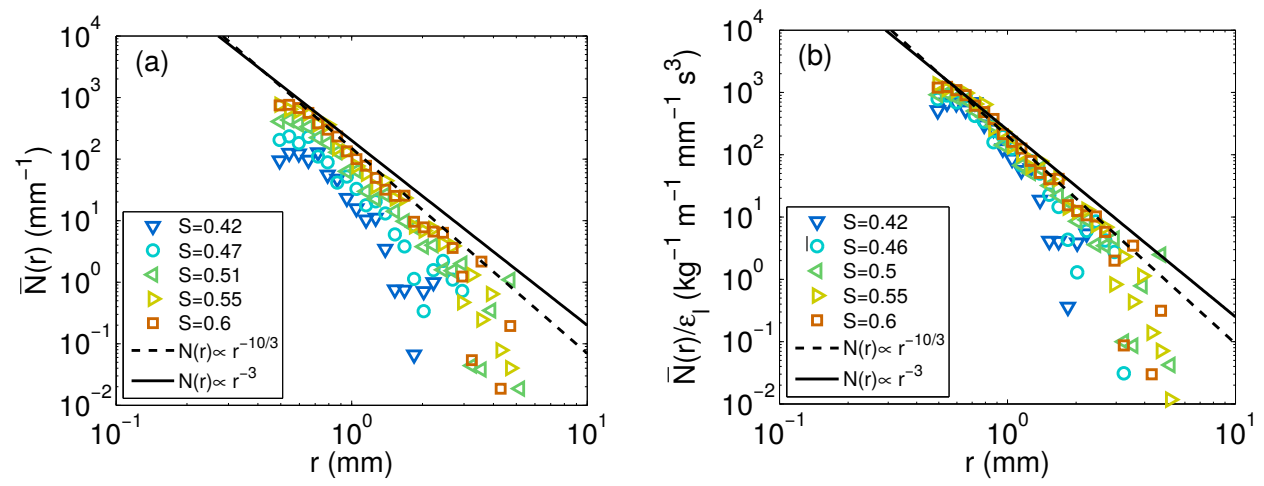

Figure 9: Ensemble-averaged data for 5 breaking waves with initial slopes from $S=0.42$ to $S=0.6$. Time-averaged bubble size distribution $\bar{N}(r)$ over the active breaking time $\tau_{b}$. (a) $\bar{N}(r), S$ (color scaled) increasing from bottom to top. The number of bubbles increases with $S$. (b) Rescaled bubble size distribution $\bar{N}(r) / \epsilon_{l}$. The data collapse significantly between (a) and (b); except for the smaller slope where the inertial breakup subrange is of limited extent. In both plots, the dashed line is $\bar{N}(r) \propto r^{-10 / 3}$ and the solid line is $\bar{N}(r) \propto r^{-3}$.

wide range of initial slopes and for roughly one decade in radii. Moreover the bubble size distribution scales with the turbulent dissipation rate, both when considering the time dependent bubble size distribution $N(r, t) \propto \varepsilon$ and the time averaged data $\bar{N}(r) \propto \epsilon_{l}$, where $\epsilon_{l}=\rho A \bar{\varepsilon}$. While the constant- $Q$ model of Garrett et al. (2000) describes the $r^{-m}$ scaling it does not quantify $Q$ in terms of the other variables, and is independent of time. In the next section $\S 4$, we discuss a new model that is consistent with the results of the DNS data.

\section{A model for bubble size distribution}

Here, we propose a new model to describe both the time dependent bubble size distribution during the active breaking stage and the time-averaged results, combining ideas from the two models described in the Introduction and the results of the DNS.

\subsection{Global balance between turbulent dissipation and work done against buoyancy forces}

When a wave breaks, it entrains a tube of air of cross sectional area $A \approx \pi h^{2} / 4$, displayed in Figure 10, creating a turbulent bubble cloud over a similar area for short times after the breaking onset. Here $h$ is the vertical distance between the crest of the breaker and its point of impact on the surface below and we assume geometrical similarity for the area $A$ across the range of wave slopes. We aim to describe the number of bubbles and their size distribution within this area. The bubble size distribution per unit volume $\mathcal{N}(r, t)$ is then related to the absolute bubble size distribution $N(r, t)$ by

$$
N(r, t)=V_{0} \mathcal{N}(r, t)=A L_{c} \mathcal{N}(r, t),
$$

with $V_{0}=A L_{c}=\pi h^{2} L_{c} / 4$ the maximum volume of entrained air during breaking and $L_{c}$ the length of breaking crest.

The core of the model is to use the simple assumption based on empirical data (Lamarre \& Melville 1991; Blenkinsopp \& Chaplin 2007; Lim et al. 2015) that globally (integrating over space and time of the event) the work done against buoyancy forces in entraining 


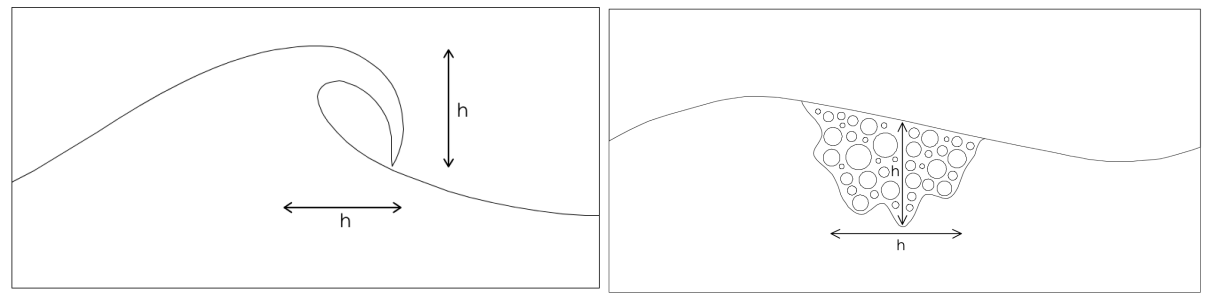

Figure 10: Sketch of the cross sectional area of the air entrainment by a breaking wave: a tube of air of section $A \propto h^{2}$ is entrained (left) and creates a turbulent bubble cloud over a similar area (right).

the bubbles is proportional to the mechanical energy dissipated. This statement can be represented by the following equation:

$$
\iiint \rho g \mathcal{N}(r, \mathbf{x}, t) \frac{4 \pi}{3} r^{3} w(\mathbf{x}, t) d r d \mathbf{x} d t=B \iint \rho(1-\alpha(\mathbf{x}, t)) \varepsilon(\mathbf{x}, t) d \mathbf{x} d t,
$$

where $\varepsilon(\mathbf{x}, t)$ is the local dissipation rate in the water, $w(\mathbf{x}, \mathbf{t})$ the local vertical velocity of the bubble cloud and $\alpha(\mathbf{x}, t)$ the void fraction (volume of air per unit volume), all functions of space $\mathbf{x}$ and time $t$, and $B$ is a dimensionless constant.

Recall that $\varepsilon(\mathbf{x}, t)$ is the local viscous dissipation rate, $\varepsilon(\mathbf{x}, t)=2 \nu D_{i j} D_{i j}$, with $D_{i j} \equiv$ $\left(\partial_{i} u_{j}+\partial_{j} u_{i}\right) / 2$ (Pope 2000). Therefore the total mechanical energy dissipated in the water is the integral over the volume of water and time $\iint \rho(1-\alpha(\mathbf{x}, t)) \varepsilon(\mathbf{x}, t) d \mathbf{x} d t$, where the inclusion of the void fraction in the equation ensures the integration in the water only.

We first assume, for simplicity, that $\alpha \ll 1$, and that the density of water $\rho$ is constant, so that Eq. 4.2 becomes:

$$
\iiint g \mathcal{N}(r, \mathbf{x}, t) \frac{4 \pi}{3} r^{3} w(\mathbf{x}, t) d r d \mathbf{x} d t=B \iint \varepsilon(\mathbf{x}, t) d \mathbf{x} d t .
$$

Now let us assume that locally, the bubble size distribution is proportional to the turbulent dissipation rate, i.e. $\mathcal{N} \propto \varepsilon$, which is supported by the DNS data (Figure 7). The time-lag between air entrainment and dissipation observed in the DNS results can be incorporated at this stage by setting, $\mathcal{N} \propto \varepsilon(t-\Delta \tau)$; c.f. Figure 7 .

With $\mathcal{N} \propto \varepsilon(t-\Delta \tau)$, we now assume separation of variables to give

$$
\mathcal{N}(r, \mathbf{x}, t)=R\left(r, r_{m}\right) \varepsilon(\mathbf{x}, t-\Delta \tau)
$$

where $r_{m}$ is the maximum bubble size, then Eq. 4.3 becomes:

$$
\int^{r_{m}} g R\left(r, r_{m}\right) \frac{4 \pi}{3} r^{3} d r \iint \varepsilon(\mathbf{x}, t-\Delta \tau) w(\mathbf{x}, t) d \mathbf{x} d t=B \iint \varepsilon(\mathbf{x}, t) d \mathbf{x} d t .
$$

Therefore:

$$
\int^{r_{m}} g R\left(r, r_{m}\right) \frac{4 \pi}{3} r^{3} d r=B W^{-1}, \text { with } W=\frac{\iint w(\mathbf{x}, t) \varepsilon(\mathbf{x}, t-\Delta \tau) d \mathbf{x} d t}{\iint \varepsilon(\mathbf{x}, t) d \mathbf{x} d t},
$$

where $W$ is the dissipation-weighted vertical mean velocity of the bubble plume over 
the active breaking period. For any breaking event, $W$ and $r_{m}$ are constants, and play parametric roles in further development of the model.

$W$ is the weighted vertical velocity of the bubble cloud and corresponds to an average rise velocity of the bubble plume, so that together with gravity, it can be interpreted as the variable corresponding to the bubble buoyancy forces. Taking into account $r_{m}$ in the dimensional analysis corresponds to considering the role of the maximum bubble size in the fragmentation process.

Now, Eq. 4.6 dimensionaly constrains the scaling with respect to $W$ and $g$, so that,

$$
\mathcal{N}(r, \mathbf{x}, t) \propto B \frac{\varepsilon(\mathbf{x}, t-\Delta \tau)}{W g} f\left(r, r_{m}\right),
$$

where $R\left(r, r_{m}\right) \propto B f\left(r, r_{m}\right) /(W g)$. Seeking power law solutions, with $f\left(r, r_{m}\right)$ having the dimensions of $\mathcal{N},\left[L^{-4}\right]$, we have,

$$
\mathcal{N}(r, \mathbf{x}, t)=B \frac{3(4-m)}{4 \pi} \frac{\varepsilon(\mathbf{x}, t-\Delta \tau)}{W g} r^{-m} r_{m}^{m-4},
$$

with $m<4$ to avoid divergence of Eq. 4.6. The $(4-m)$ constant is introduced to be consistent with the initial equation, Eq. 4.2 and cancel the numerical constant when integrating over all bubble radii. Note also that available experimental data and our numerical data suggest $3<m<4$. At this point, we have a constraint on the prefactor of the bubble size distribution, i.e. on the total volume of air, but we need information on the statistics to determine $m$.

Equation 4.8 can be justified by a dimensional analysis. Say $\mathcal{N}$ depends on the bubble scales, i.e. the radius $r$ and the radius of the initial largest bubble $r_{m}$; the buoyancy forces, i.e. gravity $g$ and the weighted bubble rise velocity $W$; and the turbulent dissipation rate $\varepsilon$ that is responsible for the break-up. At this point we neglect the surface tension, since we consider only bubbles that can be fragmented by turbulence and therefore are larger than the Hinze scale (following Garrett et al. (2000) and Deane \& Stokes (2002)).

Thus we have 6 variables and 2 dimensions, so a relationship can be written between 4 dimensionless variables:

$$
\mathcal{N} r_{m}^{4}=G\left(\frac{r}{r_{m}}, \frac{\varepsilon}{g W}, \frac{W^{2}}{g r_{m}}\right) .
$$

Within the turbulent inertial subrange of the bubble cascade, experimental and numerical data show that $0.1 \leqslant r / r_{m} \leqslant 1$ (see $\S 5$ ). The ratio between turbulence and buoyancy forces can be estimated using the numerical data and is typically $0.1 \leqslant \frac{\varepsilon}{g W} \leqslant 1$ (see $\S 5)$. The last dimensionless number can be estimated as follow. As discussed in $\S 5, W$ is $O(10) \mathrm{cm} / \mathrm{s}$, and available experimental data suggest $r_{m}$ is $O(1-10) \mathrm{cm}$, so that $W^{2} /\left(g r_{m}\right)$ is $O\left(10^{-2}-10^{-1}\right)$. Therefore $W^{2} /\left(g r_{m}\right) \ll 1$, reaching the smallest values of the dimensionless variables. Following the common assumption of asymptotic independence, we neglect this variable in the subsequent analysis and ultimately test its neglect in the comparison with the available data and our numerical results (see $\S 5$ ). Note that other dimensionless numbers could have been proposed, but they all are combinations of those in Eq. 4.9. For example, $W^{3} /\left(\varepsilon r_{m}\right)=(\varepsilon / g W)^{-1} \times W^{2} /\left(g r_{m}\right)$ and we also find $W^{3} /\left(\varepsilon r_{m}\right)$ is $O\left(10^{-2}-10^{-1}\right)$, and can be neglected in the same way.

Seeking power-law solutions, this leads to

$$
\mathcal{N} r_{m}^{4} \propto\left(\frac{r}{r_{m}}\right)^{-m}\left(\frac{\varepsilon}{g W}\right)^{p}
$$


Now $p=1$ is determined experimentally, equivalent to $\mathcal{N} \sim \varepsilon$. It leads to

$$
\mathcal{N} \propto \frac{\varepsilon}{g W}\left(\frac{r}{r_{m}}\right)^{-m} r_{m}^{-4}
$$

which is equivalent to Eq. 4.8.

Note that we can relax our assumption that $\alpha \ll 1$, so that $W$ becomes:

$$
W=\frac{\iint \varepsilon(\mathbf{x}, t-\Delta \tau) w(\mathbf{x}, t) d \mathbf{x} d t}{\iint(1-\alpha(\mathbf{x}, t)) \varepsilon(\mathbf{x}, t) d \mathbf{x} d t}
$$

which is a slightly more complicated dissipation-weighted vertical velocity, characterizing the active breaking event. This does not change the dimensional analysis and the bubble size distribution per unit volume is still given by Eq. 4.8.

\subsection{Adapting the dimensional analysis from Garrett et al. (2000)}

To determine the value of $m$, we recall the dimensional analyis from Garrett et al. (2000). As discussed in the Introduction, it considers that air is initially injected into large bubbles, and these are then broken up into smaller ones by turbulent velocity fluctuations. Then, it assumes that the inflow of air has no back effect on the turbulence, so that the bubble size distibution is proportional to the average air flow rate $Q$ (per unit time and per unit volume of water), and only depends on the bubble radius $r$ and the turbulence dissipation rate $\varepsilon$. Dimensional analysis leads to

$$
\mathcal{N}(r) \propto Q \varepsilon^{-1 / 3} r^{-10 / 3} .
$$

Note that the $r^{-10 / 3}$ scaling can also be justified by a mechanistic sequential break-up argument (Garrett et al. 2000).

We now need to connect the average air flow rate $Q$ to the other variables of the problem. The time of air injection can be estimated by the time to create the bubble cascade, i.e. the time to fragment bubbles from the largest bubbles in the system $r_{m}$ to bubbles close to the Hinze scale. The fragmentation time, or lifetime of a bubble of radius $r, \tau(r)$ is given by the ratio of the size of the bubble $r$ and the turbulent velocity fluctuations at this scale $\Delta v \sim(\varepsilon r)^{1 / 3}$ (Martinez-Bazan et al. 1999; Garrett et al. 2000)

$$
\tau(r) \sim r(\varepsilon r)^{-1 / 3} \sim r^{2 / 3} \varepsilon^{-1 / 3} .
$$

Say $q$ successive fragmentations are needed, each bubble giving $n$ bubbles (as in the sequential mechanistic break-up argument used in Garrett et al. (2000)), then the total time to create the cascade is

$$
\begin{aligned}
\tau_{c} & =\tau_{1}+\tau_{2}+\ldots=\tau\left(r_{m}\right)+\sum_{i=2}^{q} \tau_{i}\left(r_{i}\right) \\
& =r_{m}^{2 / 3} \varepsilon^{-1 / 3}+\sum_{i=2}^{q} r_{i}^{2 / 3} \varepsilon^{-1 / 3} \\
& =r_{m}^{2 / 3} \varepsilon^{-1 / 3}\left(1+\sum_{j=1}^{q-1}\left(n^{-1 / 3}\right)^{j-1}\right), \text { where } j=i-1, \\
& =r_{m}^{2 / 3} \varepsilon^{-1 / 3} c_{q, n}, \text { with } c_{q, n}=\left(1+\frac{n^{-(q-1) / 3}-1}{n^{-1 / 3}-1}\right),
\end{aligned}
$$

which scales as $r_{m}^{2 / 3} \varepsilon^{-1 / 3}$, so that different values of $q$ and $n$ only change the numerical 
constant in Eq. (4.15), $c_{q, n}$. Typically, for binary break-up $(n=2)$, starting with $r_{m} \approx 10$ $\mathrm{mm}, q \approx 12$ succesives break-ups are needed to reach the Hinze scale of $\approx 1 \mathrm{~mm}$, leading to a numerical prefactor of $c_{q, n} \approx 6$, whereas for a cubic cleavage of bubbles, $q=8$ the prefactor would be $c_{q, n} \approx 4$. Note that $\tau_{c}$ gives a reasonable estimation of the time-lag $\Delta \tau$ observed between the time of the maximum air entrainment and the time of the maximum observed dissipation rate.

This defines $Q$ as

$$
Q=\frac{V_{a}}{V_{w}} \frac{1}{\tau_{c}}=c_{q, n} \frac{V_{a}}{V_{w}} r_{m}^{-2 / 3} \varepsilon^{1 / 3},
$$

where $V_{a}$ is the volume of air and $V_{w}$ the volume of water, it leads to,

$$
\mathcal{N}(r) \propto \frac{V_{a}}{V_{w}} r_{m}^{-2 / 3} r^{-10 / 3} .
$$

This above argument provides information on the bubble statistics but it does not constrain the volume of air injected by the breaking wave.

Now, we insert the $m=10 / 3$ solution from Eq. 4.8, and we obtain

$$
\mathcal{N}(r, \mathbf{x}, t)=B \frac{1}{2 \pi} \frac{\varepsilon(\mathbf{x}, t-\Delta \tau)}{W g} r^{-10 / 3} r_{m}^{-2 / 3} .
$$

Once integrated in space and time over the breaking event, Eq. 4.18 and Eq. 4.17 should be equivalent, and an encouraging point is that the scaling for $r_{m}$ is indeed the same in both formulations, so finally we arrive at the following balance

$$
\frac{V_{a}}{V_{w}} \propto \frac{\varepsilon}{W g},
$$

which can be written as $V_{a} W g \propto V_{w} \varepsilon$, meaning that the spatially averaged mechanical turbulent dissipation scales with averaged buoyancy force, which was our initial assumption.

\subsection{Volume integrated bubble size distribution}

Starting from Eq. 4.18, we can now consider the absolute bubble size distribution, $N(r, t)$, over the control volume of breaking, $V_{0}=A L_{c}$, which leads to

$$
N(r, t)=A L_{c}\langle\mathcal{N}(r, \mathbf{x}, t)\rangle=B \frac{A L_{c}}{2 \pi} \frac{\varepsilon(t-\Delta \tau)}{W g} r^{-10 / 3} r_{m}^{-2 / 3},
$$

where $\langle$.$\rangle denotes spatial averaging and \varepsilon(t)=\langle\varepsilon(\mathbf{x}, t)\rangle$ the time dependent but spatially averaged dissipation rate.

The time (and space) averaged bubble size distribution $\bar{N}(r)$ is then:

$$
\bar{N}(r)=B \frac{1}{2 \pi} \frac{L_{c} A \bar{\varepsilon}}{W g} r^{-10 / 3} r_{m}^{-2 / 3}=B \frac{1}{2 \pi} \frac{L_{c} \epsilon_{l}}{\rho W g} r^{-10 / 3} r_{m}^{-2 / 3},
$$

with $\bar{\varepsilon}=\bar{\varepsilon}(t)$ the space and time averaged dissipation rate over the active breaking event, and we recall that $\epsilon_{l}=\rho A \bar{\varepsilon}$ is the dissipation rate per unit length of breaking crest.

The time and space-averaged bubble size distribution $\bar{N}(r)$ is equivalent to the bubble size distribution considered by Garrett et al. (2000) and measured experimentally by various authors (Loewen et al. 1996; Deane \& Stokes 2002; Rojas \& Loewen 2007; Blenkinsopp \& Chaplin 2010) if one assumes the bubble plume to be homogeneous over the volume $V_{0}$. Note that the adiabatic size distribution Eq. 4.20 is valid during the active breaking stage, and interestingly is a way to experimentally quantify the active time of breaking $\tau_{b}$. 
Note that, following Garrett et al. (2000), we have assumed that the bubble breakup process is independent of surface tension. Therefore, the model is valid until surface tension prevents further bubble break-up, i.e. up to the Hinze scale (Eq. 1.2), and is valid only during the active breaking stages. At later stages, once the turbulence becomes weaker, we expect the bubble distribution to decay due to bubbles rising back to the surface and bursting, and through bubble dissolution.

The model uses the dimensionally constrained power law $\bar{N}(r) \propto r^{-10 / 3}$ which is consistent with experimental observations, together with $N(r, t) \propto \varepsilon(t-\Delta \tau)$ and $\bar{N}(r) \propto$ $\epsilon_{l}$ which is consistent with our previous conjecture: when the breaking strength increases, $\varepsilon$ increases and so does the number of bubbles.

This formulation gives information on the time evolution of the bubble plume (growth and decay) in the sense that it says that it follows the time evolution of the turbulent dissipation rate. We believe that this should be of significant interest for field measurements since it could be tested if the bubble size distribution and $\varepsilon$ could be measured in dense bubble clouds.

As shown in $\S 3$, DNS results are in good agreement with this model (based in part on the DNS) for both the time-dependent and time-averaged formulations and justify the assumption that the bubble size distribution is linearly proportional to the turbulent dissipation rate $\varepsilon$.

\subsection{Derivation of the volume}

We will now use the model to scale the total volume of entrained air during the breaking process as a function of the external parameters of the waves, the initial wave slope $S$ and the related dissipation. Indeed, the model can be used to predict such scaling, since the volume of air entrained is related to the bubble size distribution by

$$
V(t)=\int_{0}^{r_{m}} \frac{4 \pi}{3} r^{3} N(r, t) d r
$$

for the time-dependent volume of entrained air and

$$
\bar{V}=\int_{0}^{r_{m}} \frac{4 \pi}{3} r^{3} \bar{N}(r) d r
$$

for the time-averaged volume of air.

Using Eq. 4.20 and 4.22, the time-dependent volume of air entrained is then given by

$$
V(t)=\int_{0}^{r_{m}} \frac{4 \pi}{3} r^{3} B \frac{A L_{c}}{2 \pi} \frac{\varepsilon(t-\Delta \tau)}{W g} r^{-10 / 3} r_{m}^{-2 / 3} d r=B \frac{\pi h^{2} L_{c}}{4} \frac{\varepsilon(t-\Delta \tau)}{W g} .
$$

The complete equations from our model for the time-averaged volume of entrained air as a function of the slope or the dissipation rate per unit length of breaking crest is then obtained from Eq. 4.23, and Eq. 4.21, so that

$$
\bar{V}=B \frac{\epsilon_{l} L_{c}}{\rho W g} .
$$

then using the inertial scaling from Drazen et al. (2008), $\epsilon_{l}=\Xi \rho g^{3 / 2} h^{5 / 2} \pi / \sqrt{2}$, with $\Xi$ an order one constant, and the linear phase speed for gravity waves, $c=\sqrt{g / k}$, we obtain

$$
\bar{V}=B \frac{\pi \Xi}{\sqrt{2}} \frac{L_{c} g^{1 / 2} h^{5 / 2}}{W},
$$

The latter equation can also be written using the relationship for $\epsilon_{l}=b \rho c^{5} / g$, and leads 
to

$$
\bar{V}=B b \frac{L_{c} c^{5}}{W g^{2}}
$$

and the breaking strength $b$ is then given by the semi-empirical formulation from Romero et al. (2012) (Eq. 3.4: $\left.b=0.4(S-0.08)^{5 / 2}\right)$

$$
\bar{V}=0.4(S-0.08)^{5 / 2} B \frac{L_{c} c^{5}}{W g^{2}} .
$$

We will now test our model against our numerical data and existing laboratory experiments.

\section{Discussion on the scaling of the volume and estimation of the bubble cloud constant $B$}

In this Section, we test our model against our DNS results and available laboratory data. We have to take into account the other variables in Eq. 4.25, i.e. mainly the weighted velocity since the gravity and the liquid density will remain constant in the breaking water wave problem.

\subsection{Weighted velocity of the bubble plume}

Now, we estimate the last unknown in our model, the weighted velocity $W$ : the weighted velocity of the bubble plume that can be calculated explicitely in our DNS from Eq. 4.12.

Figure 11 shows an example at a given time of two-dimensional maps (data have been averaged over y (i.e. for a field $\psi(x, y, z, t)$, we obtain the transversally integrated field $\left.\psi(x, z, t)=1 / L_{c} \int_{-L_{c} / 2}^{L_{c} / 2} \psi(x, y, z, t) d y\right)$, of the variables necessary to calculate $W$.

Figure 11a shows $[1-\alpha(x, z, t)]$, Figure $11 \mathrm{~b}$ shows $\varepsilon(x, z, t)$ and Figure $11 \mathrm{c}$ shows $w(x, z, t) \varepsilon(x, z, t)$. It is clear that regions of high local dissipation $\varepsilon$ correspond to regions with high void fraction, which qualitatively corroborates our assumption of local proportionality between the dissipation and the bubble population. Note that similar features are described in a recent laboratory study, that measured both the void fraction and the velocity fields under a breaking wave (Lim et al. 2015).

Figure 11d shows the weighted velocity, calculated from Eq. 4.12

$$
W=\frac{\int w(x, z, t) \varepsilon(x, z, t-\Delta \tau)(1-\alpha(x, z, t)) d t d x d z}{\left.\int \varepsilon(x, z, t)\right) d t d x d z}
$$

as a function of the wave slope. Small variations in the value of $W$ are observed for our range of parameters.

Now, we want to compare $W$ with the rise velocity of a bubble of typical size in the bubble plume during the active breaking stage. The typical bubble radius $\bar{r}$ in the bubble plume is estimated by

$$
\bar{r}=\frac{\int r \bar{N}(r) d r}{\int \bar{N}(r) d r}
$$

within the bubble inertial subrange.

The typical rise velocity is then obtained from Eq. 3.8, $\bar{w}_{b}=w_{b}(\bar{r})$ and is approximatively the weighted velocity $W$ obtained from Eq. 4.12 as shown by Figure 11 d. Note that we also find a linear relation between $W$ and the typical velocity $h / \tau_{b}$ built from the height of the breaker $h$ and the active breaking time $\tau_{b}$.

To summarize, the weighted velocity correponds to a spatially-integrated rise velocity 

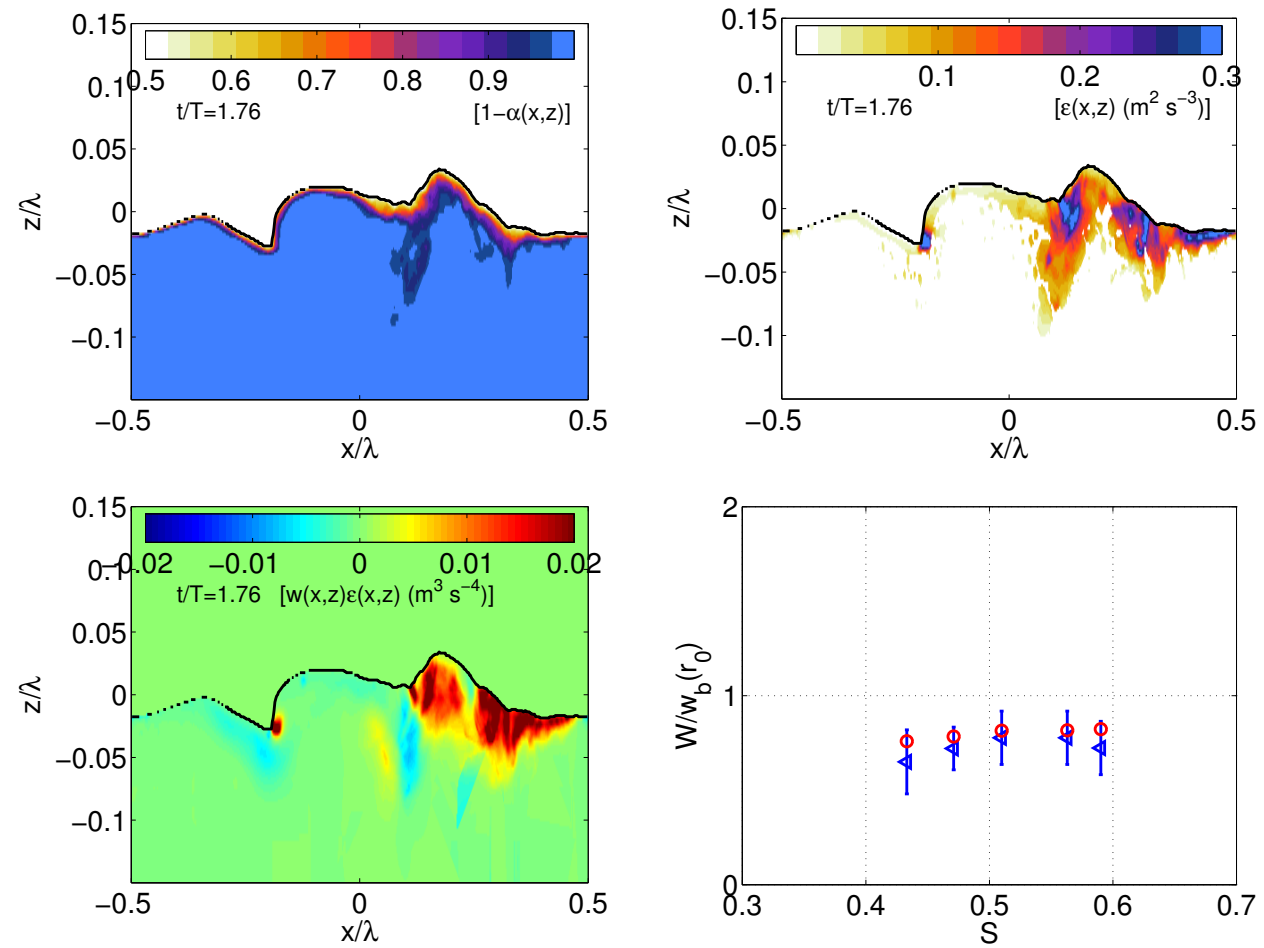

Figure 11: Example of transversally-averaged maps (in the water) of (a) [1 $\alpha(x, z, t)]$, (b) $\varepsilon(x, z, t)$, and (c) $w(x, z, t) \varepsilon(x, z, t)$ at $t / T=1.76$ as a function of $x$ and $z$, for $S=0.55$. These are used to calculate the weighted velocity $W=$ $\int w(x, z, t) \varepsilon(x, z, t) d t d x d z /\left(\int \varepsilon(x, z)(1-\alpha(x, z, t)) d t d x d z\right)$ (Eq. 4.12). (d) Weighted velocity as a function of the initial wave slope, normalized by $w_{b}\left(r_{0}=1 \mathrm{~mm}\right)$ the rise velocity in clean water of a bubble of radius $r_{0}=1 \mathrm{~mm} .(\triangleleft)$ : direct calculation of $W$ from Eq. 4.12. (०): $\bar{w}_{b}=w_{b}(\bar{r})$, the rise velocity in clean water from Eq. 3.8 of a bubble with a radius given by $\bar{r}=\int r \bar{N}(r) d r / \int \bar{N}(r) d r$.

of the bubble plume that can be successfully estimated from the rise velocity of a bubble of mean size in the plume.

\subsection{Time dependent volume of entrained air and the dissipation rate}

We have seen in Figure $7 \mathrm{f}$ that $N(r, t) \propto \varepsilon(t-\Delta \tau)$ and used that result to build our model in $\S 4$. Now, Figure 12 shows the rescaled volume of air entrained by the breaking wave $V(t) / V_{0}$, as a function of the rescaled time-dependent dissipation, $\varepsilon(t-\Delta \tau) / W g$, as suggested by Eq. 4.24, during the active breaking time. Within the scatter of the data, we observe the expected linear relation between the volume of air and the turbulent dissipation rate, $V / V_{0}=B \varepsilon(t-\Delta \tau) / W g$. It leads to a first estimate of the constant, $B \approx 0.05$.

\subsection{Normalized bubble size distribution and bubble cloud constant}

We can now test our model for the bubble size distribution, given by Eq. 4.21. Figure 13 shows the DNS data rescaled according to Eq. 4.21. The maximum bubble size $r_{m}$ for each bubble size distribution is estimated by applying the semi empirrical formulation $\bar{N}(r)=$ $N_{0} r^{-10 / 3} \exp \left(-2\left(r / r_{m}\right)^{2}\right)$ already used for the time dependent bubble size distribution 


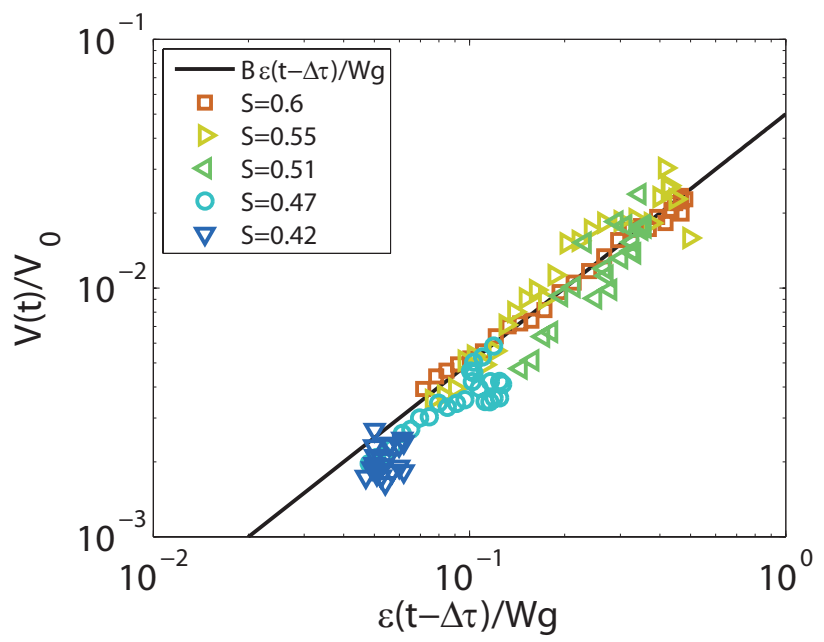

Figure 12: $V(t) / V_{0}$ as a function of $\varepsilon(t-\Delta \tau) / W g$. Color symbols are for the various initial slopes, as in Fig. 9. Solid line shows the linear relation $V / V_{0}=B \varepsilon(t-\Delta \tau) / W g$, with $B=0.05$.

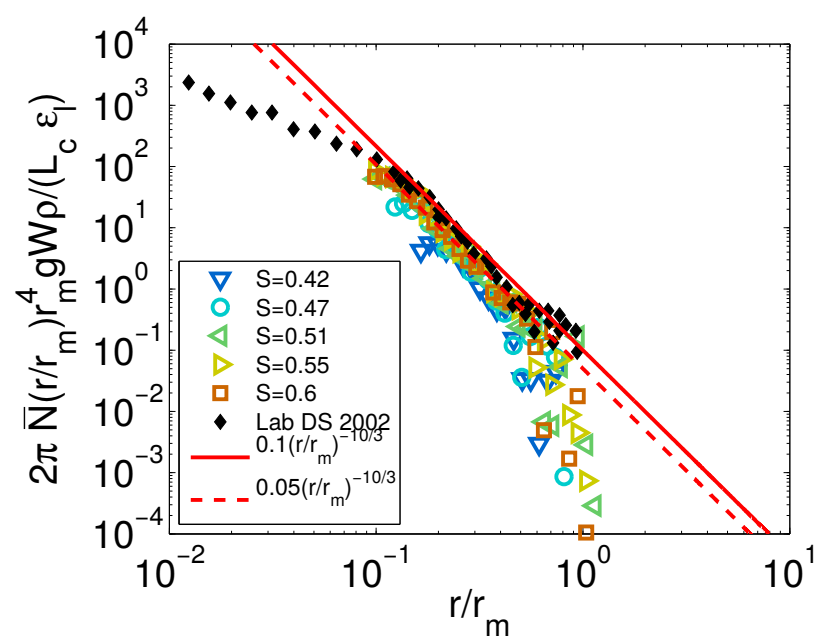

Figure 13: Rescaled bubble size distribution, according to Eq. 4.21: $\bar{N}\left(r / r_{m}\right) r_{m}^{4} g W \rho / \epsilon_{l}$, as a function of the rescaled bubble radius $r / r_{m}$. Solid line shows $B\left(r / r_{m}\right)^{-10 / 3}$, with $B=0.1$ and dashed line is $B\left(r / r_{m}\right)^{-10 / 3}$, with $B=0.05$; Color symbols are the ensembleaveraged DNS data for various slopes, as in Figure 9, with the initial slope $S$ color coded as in Figure 9. Black diamonds are laboratory experiments from Deane \& Stokes (2002).

(Figure 7 and Eq. 3.7). We find that $r_{m}$ increases with the strength of the breaking wave $S$. We obtain a good collapse of the data, as already shown in Figure 9, which was expected since the weighted velocity varies little for the range of parameters tested here (as shown in Figure 11).

We now want to compare our model with the available data from Deane \& Stokes (2002). To rescale the data from Deane \& Stokes (2002), we need to estimate the maximum bubble radius $r_{m}$, the vertical weighted velocity $W$ and the dissipation rate $\epsilon_{l}$. 
$r_{m}=10 \mathrm{~mm}$ is estimated directly from the data as the end of the bubble cascade. $\epsilon_{l}$ can be estimated from the wave packet parameters used by Deane \& Stokes (2002), the wave slope $S$, the central wavenumber and frequency, and then using the semi-empirical relationship from Romero et al. (2012), Eq. 3.4.

The remaining unknown variable is the weighted bubble plume velocity $W$. As shown by Figure 11d, $W$ can be estimated by the rise velocity of the mean bubble size of the plume using the bubble size distribution of Deane \& Stokes (2002) within the bubble cascade and the definition of the typical bubble size defined by Eq. 5.2. We obtain a typical bubble size $\bar{r}_{D S} \approx 1.5 \mathrm{~mm}$. Note that while we have compared $W$ to the rise velocity in clean water, we have here to consider the rise velocity in dirty water. The rise velocity is then given by the formula from Thorpe (1982) and Woolf \& Thorpe (1991) for a bubble rising in dirty water

$$
\begin{aligned}
w_{b}^{\text {dirty }}(r) & =\frac{2 r^{2} g}{9 \nu_{w}}\left(\left(v^{2}+2 v\right)^{1 / 2}-v\right), \text { with } \\
v & =10.82 / \chi, \text { and } \chi=\frac{g r^{3}}{\nu_{w}^{2}}
\end{aligned}
$$

which leads to $w_{b}^{\text {dirty }}\left(r_{D S}\right) \approx 13 \mathrm{~cm} / \mathrm{s}$. The uncertainty in the velocity $W$ in the laboratory data is then estimated by considering the variation of $\bar{r}_{D S}$ within the bubble cascade, i.e. radii between 1 and $5 \mathrm{~mm}$, leading to values of $w_{b}^{\text {dirty }}(r)$ between 10 and 20 $\mathrm{cm} / \mathrm{s}$.

The rescaled experimental data are then shown on Figure 13 together with the DNS data. A reasonable collapse of all data is observed within the bubble inertial subrange $0.1 \leqslant r / r_{m} \leqslant 1$. The solid and dashed lines are $B\left(r / r_{m}\right)^{-10 / 3}$, with respectively $B=0.1$ and $B=0.05$, providing an estimation of the bubble cloud constant $B$.

\subsection{Volume scaling of the DNS and available experimental data}

Now we can test our model prediction for the entrained volume of air.

Figure 14 shows the DNS data for the total volume entrained during the breaking event, where all the variables are measured, together with the available laboratory data. As discussed in the review by Kiger \& Duncan (2012), only few measurements of the entrained air exist: Lamarre \& Melville (1991) and Blenkinsopp \& Chaplin (2007). The measurements from Blenkinsopp \& Chaplin (2007) can not be used here, since wave breaking is obtained when the wave propagates over a shoal. Together with the data from Lamarre \& Melville (1991) and Deane \& Stokes (2002), we also consider the data from Duncan (1981), who studied breaking waves produced by a towed hydrofoil, and measured the breaking volume, i.e. the volume of the bubble plume, which is not directly the volume of air.

However, while all the variables are measured in the present numerical study, this is not the case in the experiments from Duncan (1981), Lamarre \& Melville (1991) and Deane \& Stokes (2002), and some assumptions are needed for the missing variables. First, for all sets of laboratory data, we use the weighted velocity estimated using the bubble size distribution from Deane \& Stokes (2002) and discussed above. The volume of air entrained in the case of Deane \& Stokes (2002) is obtained by integration of the bubble size distribution.

Lamarre \& Melville (1991) measured the volume of entrained air together with the properties of the wave packet. While the values of $\epsilon_{l}$ are not given in the original paper, it can be retrieved from the given initial parameters of the waves (slope $S$, central frequency and wavelength) and the semi-empirical formula from Romero et al. (2012) (Eq. 3.4). 

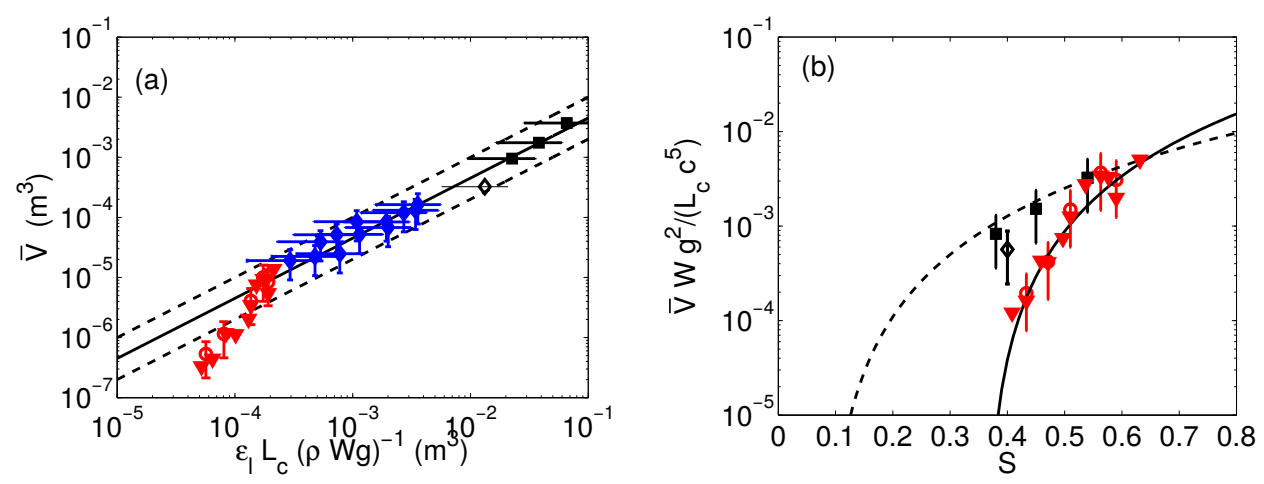

Figure 14: (a) Volume of entrained air $\bar{V}$ as a function of $\epsilon_{l} L_{c}(\rho W g)^{-1}$. (b) Normalized volume of air $\bar{V} W g^{2} /\left(L_{c} c^{5}\right)$ as a function of the initial wave slope $S$. In (a) and (b): ( $\left.\mathbf{v}\right)$ are $V=\int V(t) d t / \tau_{b}$, the total volume of air during the active breaking process for the 12 single runs and (०) for the 5 ensemble average runs. (ם) are the experimental data from Lamarre \& Melville (1991), ( $\checkmark)$ are data from Duncan (1981), and $(\diamond)$ is the data from Deane \& Stokes (2002). In (a), solid line is $V=B \epsilon_{l} \rho^{-1} L_{c}(W g)^{-1}$, with $B=0.1$, while dashed lines indicate the range of confidence of the constant $B$, ranging from 0.04 to 0.2 . In (b) solid line describes the DNS data only and is $V W g^{2} /\left(L_{c} c^{5}\right)=0.12(S-0.36)^{5 / 2}$. The dashed line is Eq. 4.28: $V W g^{2} /\left(L_{c} c^{5}\right)=0.06 \times 0.4(S-0.08)^{5 / 2}$.

In the case of Duncan (1981), $\epsilon_{l}$ is measured, together with the volume of active breaking, i.e. the volume of the air-water bubble plume observed during the spilling breaking events. To obtain the volume of air entrained, we have to multiply this measured volume by the typical average void fraction in a spilling breaker, $\bar{\alpha}=0.23 \pm 0.06$, according to recent laboratory measurements from Rojas \& Loewen (2010). Note that, in our DNS, we find a similar average void fraction within the breaking area and during the active breaking stage of a spilling breaker. The uncertainty in the mean void fraction is used as error bar in the volume of air entrained. However, while the slope at breaking could be evaluated (from the measured wave height at breaking and the wave phase speed), it would be difficult to compare with the initial slope of our DNS or the slope of the packet from Lamarre \& Melville (1991) due to the differences in the route to breaking.

When comparing the DNS, the laboratory experiments and the model, we have to keep in mind the various assumptions made to estimate the missing variables in the experiments and the related (large) error bars associated with these estimations. In the DNS, the error bars on the volume are estimated from the ensemble average data (see Appendix B) and from the two ways to estimate the total volume (direct measure $V$ and estimation through the void fraction $V_{\alpha}$ ).

Figure 14a shows the total volume of air $\bar{V}=\int V(t) d t / \tau_{b}$ entrained during the breaking process as a function of $\epsilon_{l} L_{c}(\rho W g)^{-1}$, as suggested by Eq. 4.25. As already discussed, the volume of entrained air increases with the strength of the breaking wave, i.e. $\epsilon_{l}$. Reasonable agreement is found between the DNS data and the theoretical scaling derived above (Eq. 4.25) and also with the available laboratory data from Lamarre \& Melville (1991), Duncan (1981) and Deane \& Stokes (2002). The reasonable agreement with the linear relationship predicted by our model spans several orders of magnitude, which is very encouraging, especially regarding the remaining uncertainties in the estimation of $W$ in the laboratory data. We obtain an evaluation of the bubble cloud constant, $B \approx 0.1 \pm 0.05$. 
Figure 14b shows the total volume of air normalized according Eq. 4.27, as a function of the wave slope $S$. A reasonable agreement is found between our model and the DNS data, if one includes a different breaking threshold in Eq 4.28, $\bar{V} W g^{2} /\left(L_{c} c^{5}\right)=B^{\prime}\left(S-S_{0}\right)^{5 / 2}$, with $B^{\prime}=0.5$ a constant related to $B$, the constant $\Xi$ (related to $b$ ) and the breaking threshold $S_{0}$, where $S_{0}=0.36$ in our breaking configuration. On the other hand, using the semi-empirical relation for the breaking strength $b=0.4(S-0.08)^{5 / 2}$, where 0.08 is the experimental breaking threshold leads to $\bar{V} W g^{2} /\left(L_{c} c^{5}\right)=0.4 B(S-0.08)^{5 / 2}$, with $B=0.1$ describing reasonably well the lab data and the DNS data for high plunging breakers only.

The differences in the entrained volume of air between the DNS data and the lab data are related to two effects. First, the critical slope to trigger breaking depends on the initial conditions and the route to breaking (e.g. wave focusing, modulation instability), and the Stokes waves used here have a breaking threshold higher than the one observed in laboratory experiments using focusing wave packet. Second, the low values of the entrained air in the DNS for small slopes can also be related to the relatively short wavelength of our breaking wave, $\lambda=0.24$, and the influence of surface tension in the shape of the breaking wave, which reduces the amount of entrained air, as discussed in Song \& Sirviente (2004); Liu \& Duncan (2003, 2006); Kiger \& Duncan (2012).

We obtain a value for $B$ between 0.05 and 0.15 , which corresponds to an estimation of the ratio between the totally dissipated energy by breaking and the potential energy in the bubbles being between 5 and $15 \%$. This covers the latest estimation from the laboratory by Blenkinsopp \& Chaplin (2007) who obtain values between 5 to $10 \%$, and close to Lim et al. (2015) who reports a value of $18 \%$ for a strong plunging breaker; while earlier measurements by Lamarre \& Melville (1991) discussed values between 30 to 50 $\%$.

\section{Conclusions}

We have performed novel DNS of the two-phase air-water flow in three-dimensional breaking waves, to investigate the time evolution of the entrained air. The DNS results regarding the energy budget of the breaking wave, the void fraction values during the active breaking stages, the time-averaged bubble size distribution, and the time evolution of the total volume of air, when compared with the available data support the use of the DNS to reproduce accurately the physics of air entrainment by breaking waves.

Based on the DNS results, we propose a phenomenological model for the bubble size distribution based on the assumption that the dissipated energy during breaking scales with the work done against buoyancy forces to entrain air, as well as turbulent break-up model from Garrett et al. (2000). This extended model describes the time evolution of the bubble cloud during the active breaking stage in that it relates the bubble size distribution to the instantaneous turbulent dissipation rate. In the same spirit, it describes the time-averaged bubble size distribution for one breaking event. From the bubble size distribution, the total volume of air entrained can be physically scaled to the energy dissipated due to breaking and to the characteristic wave slope at breaking. Within the scatter of the data, the model is consistent with the DNS and the available experimental data.

This numerical modeling study of entrainment by breaking waves has shown that the prospects are strong for being able to follow the example of Romero et al. (2012) who modeled the dynamics of breaking using the inertial scaling of dissipation due to breaking (Drazen et al. 2008) along with field measurements of the kinematics to improve ocean wave modeling. In this case, we foresee the possibility of using the results of this paper, 

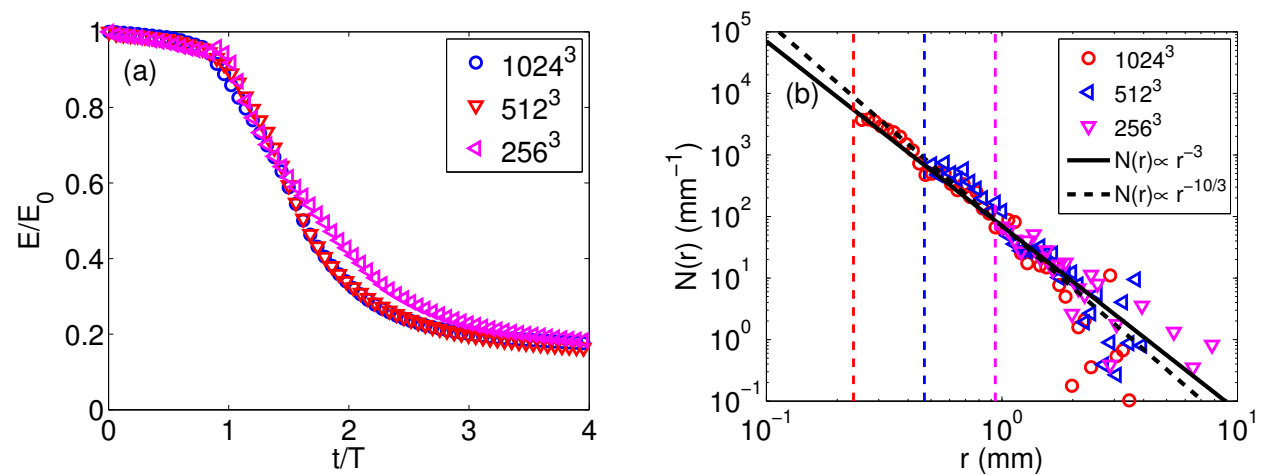

Figure 15: Convergence study for three mesh sizes, $1024^{3}(\circ), 512^{3}(\triangleleft)$ and $256^{3}(\nabla)$, for a plunging breaker $(S=0.55, B o=200, R e=40000)$. (a) Normalized energy as a function of time. The energy evolution converges with the resolution between the grid size $512^{3}$ and $1024^{3}$. (b) Time averaged bubble size distribution $\bar{N}(r)$. Vertical dashed lines indicate the minimum grid size for each case. For bubbles larger than the grid scale, $\bar{N}(r)$ is unchanged.

along with field measurements of breaking to improve the models of air entrainment, and ultimately air-sea gas transfer (c.f. Liang et al. (2011, 2012)).

Extensive experimental investigations of the bubble size distribution for various initial wave slopes, and therefore a broad range of turbulent dissipation rates are now required to further test the model presented here and the semi-empirical formulation for the volume of entrained air.

We are indebted to an anonymous reviewer for bringing to our attention an error in our model in the first version of the paper. We thank Nicholas Statom for Figure 10 and Nicholas Pizzo for helpful discussions. Computations were partially performed using allocation TG-OCE140023 to LD from the Extreme Science and Engineering Discovery Environment (XSEDE), which is supported by NSF grant number ACI-1053575. This research was supported by grants to WKM from NSF (OCE) and ONR (Physical Oceanography).

\section{Appendix A. Convergence of the numerical results with the mesh size}

In this section, we discuss the convergence of the numerical results with mesh size. Three simulations are considered, with effective resolution equivalent to $256^{3}, 512^{3}$ and $1024^{3}$ on a regular grid. The physical parameters of the simulations are $R e=40000$ and $B o=200$, as in the rest of the paper and we consider a plunging breaker with an initial slope of $S=0.55$.

Figure 15a shows the evolution of the normalized energy $E / E_{0}$ for the three grid sizes. While significant differences are seen between the coarse grid $256^{3}$ and the two finer grids, the $512^{3}$ and $1024^{3}$ resolutions are almost identical except for the initial decrease during breaking although the final values are almost identical. As a consequence, the dissipation rate $\epsilon_{l}$ does not depend on the resolution for grid size larger than $512^{3}$. This shows, together with the comparison of the breaking parameter in the simulations with the available laboratory data on Figure 2, that we are correctly resolving the dissipative 
scales involved in the breaking process and that results such as the dissipation rate per unit length of breaking crest are not changed when the grid resolution is increased and finer scales are resolved.

Figure 15b shows the time averaged bubble size distribution $\bar{N}(r)$ for the three grid sizes and shows that for bubbles larger than the mesh size, all three bubble size distribution are very close, with only small differences in the large bubble statistics. Thus for $r>1 \mathrm{~mm}$, the observation that $N(r) \propto r^{-m}$ with $m \in[3: 3.5]$ is independent of the mesh resolution. This turbulent break-up cascade goes to $r \approx 5 \mathrm{~mm}$. Moreover, working with a finer grid gives access to smaller bubbles created by further break-up. This fact is expected since experimentally, bubbles smaller than $0.1 \mathrm{~mm}$ can be observed (Deane \& Stokes 2002). The change of the shape of $\bar{N}(r)$ reported by Deane \& Stokes (2002) and Blenkinsopp \& Chaplin (2010) is not clearly observed in our simulations since the Hinze scale, given by Eq. 1.2, $r_{H} \approx 0.8 \mathrm{~mm}$ is too close to the size of the mesh for the two higher resolutions cases (and is not reached in the low resolution case). We also have to keep in mind the expected shape below the Hinze scale is not completely known since experimental results give different shapes (see Figure 1) and no theoretical framework exists.

In summary, while the shape around and below the Hinze scale is difficult to confirm in our simulations due to the resolution, the $r^{-m}$ power law for $r>r_{H}$ is not affected by the grid resolution we used. Moreoever, variations of the total volume are within the statistical errors found when investigating various runs with small perturbations, that are discussed in Appendix B.

In conclusion, the observation of $N(r) \sim r^{-m}$ with $m \in[3: 3.5]$ for $r>0.8 \mathrm{~mm}$ and within a bubble cascade inertial subrange is independent of the simulation mesh for a resolution of $512^{3}$ or better. Moreover, in the present DNS, using a $512^{3}$ mesh equivalent, we are resolving the dissipation in this two-phase turbulent flow. This validates our choice to work with a $512^{3}$ equivalent grid.

Finally, we have checked that the errors in mass (and therefore volume) conservation in both the air and water are not significant. Mass conservation is usually very good in Gerris simulations as discussed by Popinet (2009). In our simulations, errors in mass conservation are below $0.01 \%$, as shown in Fig. 16. Fig. 16 (left) shows the relative error of the volume of air and water as a function of time for a simulation $(S=0.55)$ with the $512^{3}$ resolution. The error remains below $0.01 \%$ for mass conservation of both the air and the water. Finally Fig. 16 (right) shows the maximum error for the three resolutions tested $\left(256^{3}, 512^{3}, 1024^{3}\right)$. As expected the error decreases when the resolution is increased, and is always smaller than $0.01 \%$, becoming as small as $0.001 \%$ in the $1024^{3}$ cases.

\section{Appendix B. Statistical noise and ensemble average}

In this section we present the details of an ensemble average of 6 plunging breakers. The physical parameters of the simulations are $R e=40000$ and $B o=200$, as in the rest of the paper, and the initial slope is $S=0.6$. As discussed in $\S 2$, we introduce tranverse perturbations in the initial conditions to obtain this ensemble of realisations of the breaking wave. The perturbation of the interface is $\eta_{p}=a_{p} \cos \left(k_{p} y\right)$, with $a_{p} \ll a$ and $k_{p} \in[2: 8] k$ with the corresponding perturbation on the velocity potential of the initial third-order Stokes waves.

Figure 17a shows the evolution of the total wave energy as a function of time for the 7 realisations. There are no visible differences between the runs, showing that small transverse perturbation has no effect on the global energy budget. Figure 17b shows the time evolution of the instantaneous dissipation rate (averaged over space) for the 

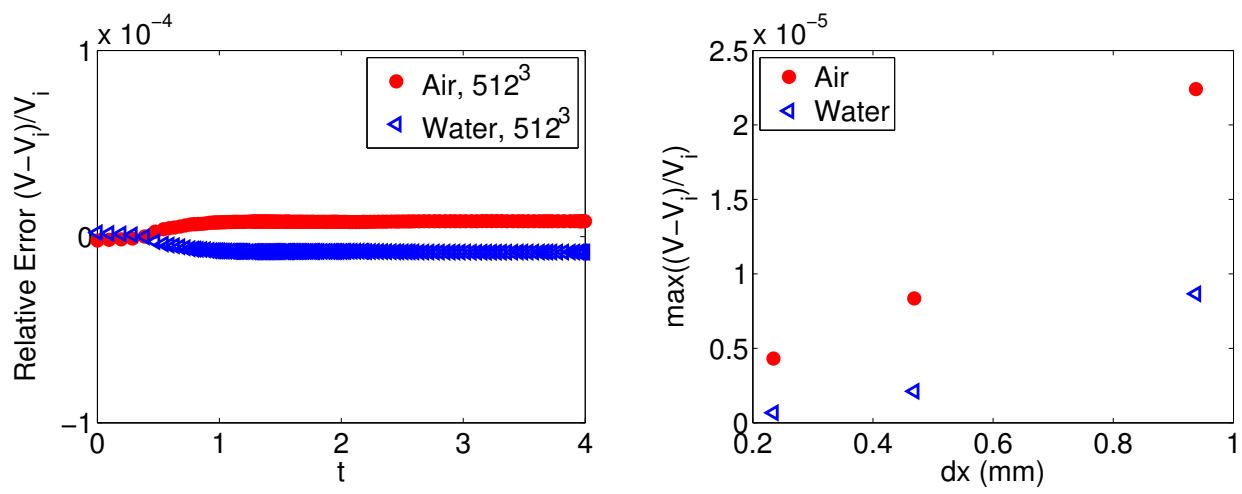

Figure 16: Mass (Volume) conservation. Left: time evolution of the relative error in volume of air and water in the numerical domain, for $S=0.55$ and the $512^{3}$ resolution. The error always remain below $0.01 \%$. Right: maximum error for the three tested resolutions. Error is below $0.001 \%$ in the $1024^{3}$ case.
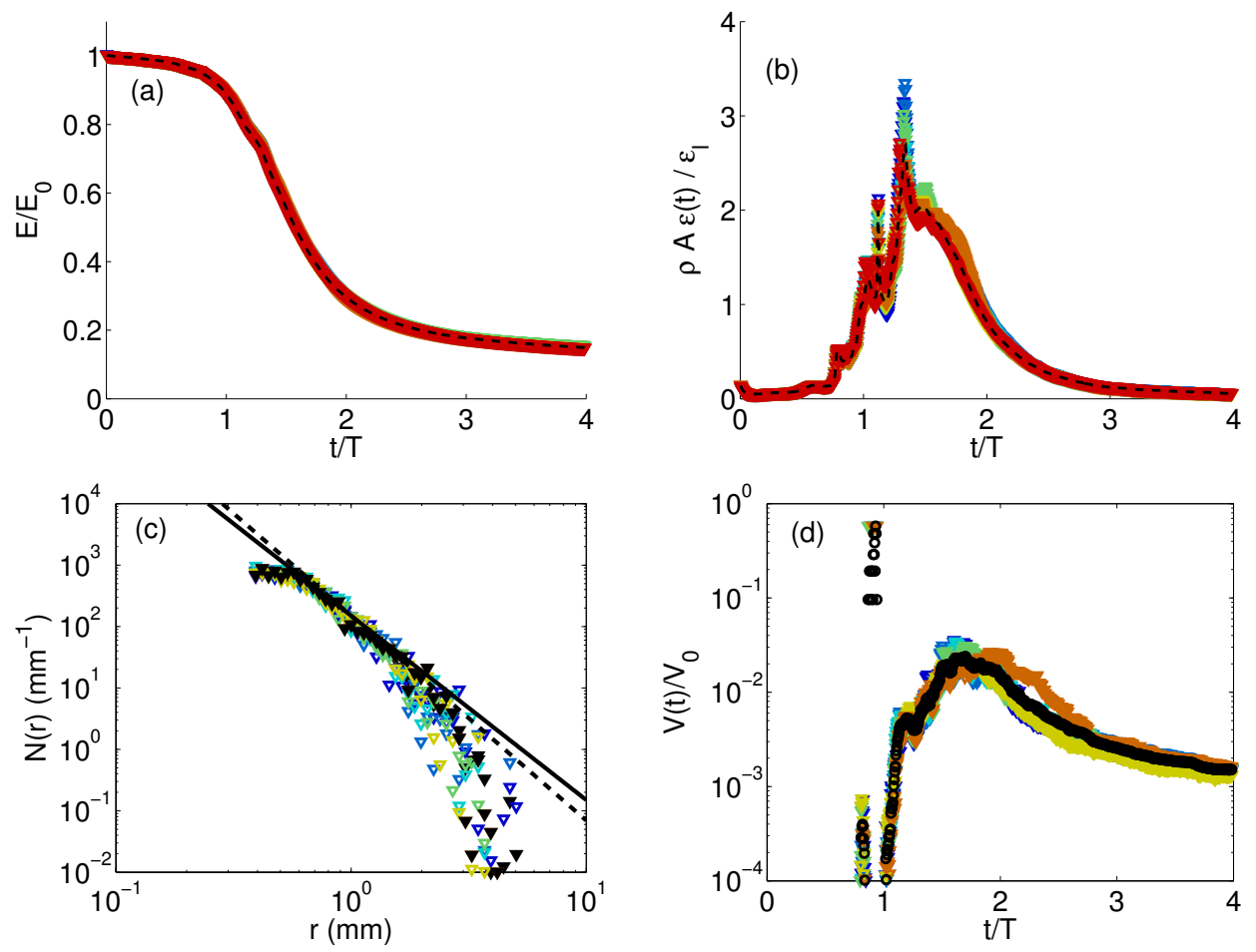

Figure 17: Ensemble average study of seven runs for a plunging breaker $(S=0.6, B o=$ 200, $R e=40000$ ). (a) Normalized energy as a function of time. There are no visible differences is the global energy decay for the various pperturbations investigated here. Black dashed lines indicate the ensemble averages in (a) and (b). (b) Normalized spatially averaged dissipation rate $\varepsilon(t) /\left(\rho A \epsilon_{l}\right)$ as a of function time. (c) Time-averaged bubble size distribution $\bar{N}(r)$. Black symbols correspond to the ensemble average. Solid line is $\bar{N}(r) \propto r^{-3}$ and dashed line is $\bar{N}(r) \propto r^{-10 / 3}$ (d) Time evolution of the entrained volume of air. Black symbols correspond to the ensemble average. 
different runs. Differences of up to $20 \%$ are observed in the amplitude of the maximum dissipation, together with small time lags between the runs.

Figure 17c shows the time-averaged bubble size distribution for each runs and the ensemble-averaged one. As expected, the scatter around the ensemble average increases with increasing bubble size and decreasing bubble number density. Figure 17d shows the time evolution of the entrained air. Again, differences appear between the runs, showing that the transverse perturbations of the breaking wave influence the air entrainment process. Typical variations of the maximum amount of air entrained and the total averaged volume of entrained air during the breaking process can be up to $50 \%$. Note that the runs with higher values of the volume also correspond to the one with higher values of the dissipation rate. Note that such variations between multiple runs in laboratory experiments are also to be expected.

Thus, multiple realisations of the same breaking wave numerical experiment show that the mean dynamical properties of the breaking wave are not too sensitive to the transverse perturbations and display the accuracy of our simulations. However, as expected, the use of ensemble-averaged data significantly reduces the statistical noise in the estimation of the bubble size distribution.

\section{REFERENCES}

Agbaglah, G., Delaux, S., Fuster, D., Hoepffner, J., Josserand, C., Popinet, S., Ray, P., Scardovelli, R. \& Zaleski, S. 2011 Parallel simulation of multiphase flows using octree adaptivity and the volume-of-fluid method. Comptes Rendus Mecanique 339 (23), $194-207$.

Andreas, E. L., Edson, J. B., Monahan, E. C., Rounult, M. P. \& Smith, S. D. 1995 The spray contribution to net evaporation from the sea: A review of recent progress. BoundaryLayer Meteorology $\mathbf{7 2}$ (1-2), 3-52.

BALDY, S. 1993 A generation-dispersion model of ambient and transient bubbles in the close vicinity of breaking waves. J. Geophys. Res. 98 (C10), 18277-18293.

Banner, M.L. \& Peirson, W. L. 2007 Wave breaking onset and strength for two-dimensional deep-water wave groups. J. Fluid Mech. 585 (1), 93-115.

Blenkinsopp, CE \& Chaplin, JR 2007 Void fraction measurements in breaking waves. Proc. R. Soc. A 463 (2088), 3151-3170.

Blenkinsopp, C. E. \& Chaplin, J. R. 2010 Bubble size measurements in breaking waves using optical fiber phase detection probes. Oceanic Engineering, IEEE Journal of $\mathbf{3 5}$ (2), 388-401.

Chen, G., Kharif, C., Zaleski, S. \& Li, J. 1999 Two dimensionnal navier stokes simulation of breaking waves. Phys. Fluid 11, 121-133.

Chen, Xiaodong, Ma, Dongunn, Yang, Vigor \& Popinet, Stephane 2013 High-fidelity simulations of impinging jet atomization. Atomization and Sprays 23 (12).

Deane, G.B. \& Stokes, M.D. 2002 Scale dependance of bubble creation mechanisms in breaking waves. Nature 418, 839-844.

Deike, L., Fuster, D., Berhanu, M. \& Falcon, E. 2014 Direct numerical simulations of capillary wave turbulence. Phys. Rev. Lett. 112, 234501.

Deike, L., Popinet, S. \& Melville, W.K. 2015 Capillary effects on wave breaking. J. Fluid Mech. 769, 541-569.

Derakhti, M. \& Kirby, J.T. 2014 Bubble entrainment and liquid-bubble interaction under unsteady breaking waves. J. Fluid Mech. 761, 464-506.

Drazen, D. A., Melville, W. K. \& Lenain, L. 2008 Inertial scaling of dissipation in unsteady breaking waves. J. Fluid Mech. 611 (1), 307-332.

DUNCAN, JH 1981 An experimental investigation of breaking waves produced by a towed hydrofoil. Proceedings of the Royal Society of London. A. Mathematical and Physical Sciences 377 (1770), 331-348. 
Farmer, D.M., McNeil, C.L. \& Johnson, B.D. 1993 Evidence for the importance of bubbles in increasing air-sea gas flux. Nature 361, 620-623.

Fuster, D., Agbaglah, G., Josserand, C., Popinet, S. \& Zaleski, S. 2009 Numerical simulation of droplets, bubbles and waves: state of the art. Fluid Dyn Res 41, 065001.

Fuster, D., Matas, J.-P, Marty, S., Popinet, S., Hoepffnner, J., Cartellier, A. \& ZALESKI, S. 2013 Instability regimes in the primary breakup region of planar coflowing sheets. J. Fluid Mech. 736, 150-176.

Garrett, C., Li, M. \& FArmer, D. 2000 The connection between bubble size spectra and energy dissipation rates in the upper ocean. J. Phys. Oceanogr. 30 (9), 2163-2171.

Gemmrich, Johannes R, Banner, Michael L \& Garrett, Chris 2008 Spectrally resolved energy dissipation rate and momentum flux of breaking waves. Journal of Physical Oceanography 38 (6), 1296-1312.

Grare, L., Peirson, W. L., Branger, H., Walker, J. W., Giovanangeli, J-P. \& Makin, V. 2013 Growth and dissipation of wind-forced, deep-water waves. J. Fluid Mech. 722, $5-50$.

Hinze, J. O. 1955 Fundamentals of the hydrodynamic mechanism of splitting in dispersion processes. AIChE Journal 1 (3), 289-295.

IAFRATI, A 2011 Energy dissipation mechanisms in wave breaking processes: Spilling and highly aerated plunging breaking events. J. Geophys. Res 116 (C7).

Kiger, Kenneth T \& Duncan, James H 2012 Air-entrainment mechanisms in plunging jets and breaking waves. An. Rev. Fluid Mech. 44, 563-596.

Kleiss, Jessica M \& Melville, W Kendall 2010 Observations of wave breaking kinematics in fetch-limited seas. Journal of Physical Oceanography 40 (12), 2575-2604.

Lamarre, E. \& Melville, W.K. 1991 Air entrainment and dissipation in breaking waves. Nature 351, 469-472.

Lamarre, Eric \& Melville, W. K. 1994 Voidfraction measurements and soundspeed fields in bubble plumes generated by breaking waves. J. Acoust. Soc. Am. 95 (3), 1317-1328.

de Leeuw, G., Andreas, E. L, Anguelova, M. D., Fairall, C. W., Lewis, E. R., O’Dowd, C., Schulz, M. \& Schwartz, S. E. 2011 Production flux of sea spray aerosol. Rev. Geophys. 49 (RG2001).

Leifer, I. \& DE Leeuw, G. 2006 Bubbles generated from wind-steepened breaking waves: 1. bubble plume bubbles. Journal of Geophysical Research: Oceans 111 (C6), c06020.

Liang, J.H., McWilliams, J. C., Sullivan, P. P. \& Baschek, B. 2011 Modeling bubbles and dissolved gases in the ocean. J. Geophys. Res. 116 (C3).

Liang, J-H., McWilliams, J.C., Sullivan, P.P. \& Baschek, B. 2012 Large eddy simulation of the bubbly ocean: New insights on subsurface bubble distribution and bubble-mediated gas transfer. J. Geophys. Res. 117 (C4).

Lim, Ho-Joon, Chang, Kuang-An, Huang, Zhi-Cheng \& Na, Byounguoon 2015 Experimental study on plunging breaking waves in deep water. Journal of Geophysical Research: Oceans 120 (3), 2007-2049.

Liu, Xinan \& Duncan, J. H. 2003 The effects of surfactants on spilling breaking waves. Nature 421, 520-523.

LiU, Xinan \& Duncan, J. H. 2006 An experimental study of surfactant effects on spilling breakers. J. Fluid Mech. 567, 433-455.

Loewen, M. R., O’Dor, M. A. \& SkAfel, M. G. 1996 Bubbles entrained by mechanically generated breaking waves. J. Geophys. Res. 101 (C9), 20759-20769.

Lubin, P \& GLOCKNer, S 2015 Numerical simulations of three-dimensional plunging breaking waves: generation and evolution of aerated vortex filaments. J. Fluid Mech. 767, 364-393.

Martinez-Bazan, C., Montanes, J.L. \& Lasheras, J.C. 1999 On the breakup of an air bubble injected into a fully developed turbulent flow. part 1. breakup frequency. J. Fluid Mech. 401, 157-182.

Melville, W.K. \& Matusov, P. 2002 Distribution of breaking waves at the ocean surface. Nature 417, 58-63.

Melville, W. K. 1996 The role of surface wave breaking in air-sea interaction. Annu. Rev. Fluid Mech. 28, 279-321.

Melville, W. K. \& Fedorov, A. V. 2015 The equilibrium dynamics and statistics of gravitycapillary waves. J. Fluid Mech. 767, 449-466. 
Memery, L. \& Merlivat, L. 1985 Modelling of gas flux through bubbles at the air-water interface. Tellus B 37 (4-5), 272-285.

Monahan, E.C. \& Dam, H.G. 2001 Bubbles: an estimate of their role in the global oceanic flux of carbon. J. Geophys. Res. 106 (C5), 9377-9383.

Phillips, O.M. 1985 Spectral and statistical properties of the equilibrium range in windgenerated gravity waves. J. Fluid Mech. 156 (1), 505-31.

Pizzo, N.E. \& Melville, W. K. 2013 Vortex generation by deep-water breaking waves. J. Fluid Mech. 734, 198-218.

Pope, Stephen B 2000 Turbulent flows. Cambridge university press.

Popinet, S. 2003 Gerris: a tree-based adaptative solver for the incompressible euler equations in complex geometries. Journal of Computational Physics 190, 572-600.

Popinet, S. 2009 An accurate adaptative solver for surface-tension-driven interfacial flows. Journal of Computational Physics 228, 5838-5866.

Rhein, M., Rintoul, S.R., Aoki, S., Campos, E., Chambers, D., Feely, R.A., Gulev, S., Johnson, G.C., Josey, S.A., Kostianoy, A., Mauritzen, C., Roemmich, D., Talley, L.D. \& WANG, F. 2013 Observations: Ocean. in: Climate change 2013: The physical science basis. contribution of working group i to the fifth assessment report of the intergovernmental panel on climate change. Tech. Rep.. [Stocker, T.F., D. Qin, G.-K. Plattner, M. Tignor, S.K. Allen, J. Boschung, A. Nauels, Y. Xia, V. Bex and P.M. Midgley (eds.)]. Cambridge University Press, Cambridge, United Kingdom and New York, NY, USA.

Rojas, G \& Loewen, MR 2007 Fiber-optic probe measurements of void fraction and bubble size distributions beneath breaking waves. Experiments in Fluids 43 (6), 895-906.

Rojas, G. \& Loewen, M. R. 2010 Void fraction measurements beneath plunging and spilling breaking waves. J. Geophys. Res. 115 (C8), 2156-2202.

Romero, L., Melville, W. K. \& Kleiss, Jessica M. 2012 Spectral energy dissipation due to surface wave breaking. J. Phys. Oceanogr 42, 1421-1441.

SCARDOVELli, R. \& ZALESKI, S. 1999 Direct numerical simulation of free-surface and interfacial flow. Ann. Rev. Fluid Mech. 31 (1), 567-603.

Shi, F., Kirby, J.T. \& MA, G. 2010 Modeling quiescent phase transport of air bubbles induced by breaking waves. Ocean Modelling 35 (1), 105-117.

Song, C. \& Sirviente, A. 2004 A numerical study of breaking waves. Phys. Fluid 16, 26-49.

Sutherland, P. \& Melville, W. K. 2013 Field measurements and scaling of ocean surface wave-breaking statistics. Geophys. Res. Lett. (40), 30743079.

Terrill, E.J., Melville, W.K. \& Stramski, Dariusz 2001 Bubble entrainment by breaking waves and their influence on optical scattering in the upper ocean. J. Geophys. Res. 106 (C8), 16815-16823.

Thomson, Jim, Gemmrich, Johannes R \& Jessup, Andrew T 2009 Geophysical Research Letters 36 (11).

Thoraval, M-J., Takehara, K., Etoh, T. G., Popinet, S., Ray, P., Josserand, C., Zaleski, S. \& Thoroddsen, S. T. 2012 von kármán vortex street within an impacting drop. Phys. Rev. Lett. 108, 264506.

Thorpe, SA 1982 On the clouds of bubbles formed by breaking wind-waves in deep water, and their role in air-sea gas transfer. Philosophical Transactions of the Royal Society of London A: Mathematical, Physical and Engineering Sciences 304 (1483), 155-210.

Veron, F. 2015 Ocean spray. Ann. Rev. Fluid Mech. 47, 507-538.

Woolf, DAvid K \& Thorpe, SA 1991 Bubbles and the air-sea exchange of gases in nearsaturation conditions. Journal of Marine Research 49 (3), 435-466. 